\title{
Architecting a System Model for Personalized Healthcare Delivery and Managed Individual Health Outcomes
}

\author{
Inas S. Khayal $\mathbb{D}^{1,2}$ and Amro M. Farid ${ }^{2,3}$ \\ ${ }^{1}$ The Dartmouth Institute, Geisel School of Medicine at Dartmouth, Lebanon, NH, USA \\ ${ }^{2}$ Department of Computer Science, Dartmouth College, Hanover, NH, USA \\ ${ }^{3}$ Thayer School of Engineering at Dartmouth, Hanover, NH, USA
}

Correspondence should be addressed to Inas S. Khayal; inas.khayal@dartmouth.edu

Received 25 February 2017; Revised 29 June 2017; Accepted 19 July 2017; Published 1 March 2018

Academic Editor: Dominik T. Matt

Copyright (C) 2018 Inas S. Khayal and Amro M. Farid. This is an open access article distributed under the Creative Commons Attribution License, which permits unrestricted use, distribution, and reproduction in any medium, provided the original work is properly cited.

\begin{abstract}
In recent years, healthcare needs have shifted from treating acute conditions to meeting an unprecedented chronic disease burden. The healthcare delivery system has structurally evolved to address two primary features of acute care: the relatively short time period, on the order of a patient encounter, and the siloed focus on organs or organ systems, thereby operationally fragmenting and providing care by organ specialty. Much more so than acute conditions, chronic disease involves multiple health factors with complex interactions between them over a prolonged period of time necessitating a healthcare delivery model that is personalized to achieve individual health outcomes. Using the current acute-based healthcare delivery system to address and provide care to patients with chronic disease has led to significant complexity in the healthcare delivery system. This presents a formidable systems' challenge where the state of the healthcare delivery system must be coordinated over many years or decades with the health state of each individual that seeks care for their chronic conditions. This paper architects a system model for personalized healthcare delivery and managed individual health outcomes. To ground the discussion, the work builds upon recent structural analysis of mass-customized production systems as an analogous system and then highlights the stochastic evolution of an individual's health state as a key distinguishing feature.
\end{abstract}

\section{Introduction}

1.1. Complexity. The National Academy of Sciences Report on Building a Better Delivery System states that "similar to the supply chains in manufacturing and other industries, the healthcare delivery system is so large and complex that it has become impossible for any individual, or even any single organization, to understand all of the details of its operations [1]." This statement elucidates three key points about the healthcare delivery system. First, that the healthcare delivery system is a supply chain. Second, that it is complex. Finally, that it has become this way, suggesting that it was not this way previously.

A supply chain here refers to a series of care services provided by the healthcare delivery system to the operand, the patient. This healthcare delivery system has evolved to become more complex and as such can be considered a complex adaptive system [2-5]. "A complex adaptive system is a collection of individual agents with freedom to act in ways that are not always totally predictable, and whose actions are interconnected so that one agent's actions changes the context for other agents" [3].

The next section describes how our healthcare system, which developed to treat acute conditions but is now burdened by the treatment of chronic disease, has grown in complexity. This complexity is due to an increase in the number of agents, their roles, and their relative position. These changes have led to the need for greater collaboration and information sharing. Finally, these agents are organized into systems of systems that are continually coevolving.

1.2. Current Healthcare Delivery System. The current healthcare delivery system organically developed to meet "one-off" acute conditions. It evolved to respond to any acute illness or injury that came through the door [6]. The focus of the 
TABLE 1: Healthcare delivery challenges for chronic conditions.

(1) First, by definition, chronic disease is described by a sequence of events that (d)evolve an individual's health state over a duration that is often far longer than any single visit to a healthcare facility.

(2) Second, the sequence of these events do logically depend on each other as described by medical science.

(3) Third, how any individual experiences this chronic condition is often entirely unique given their unique combination of social, behavioral, environmental, and biological risk factors.

(4) Finally, this chronic condition often affects many aspects of an individual's health that are often covered by disparate medical disciplines.

system is on the urgency of diagnosing and curing the physical anomalies of the individual-patient before they fall into more serious diagnoses [7]. Such acute episodes last on the order of days to weeks, where the individual-patient is considered a passive recipient of treatment [8]. This model of care comes from the biomedical model: the dominant allopathic medicine model introduced in the mid-19th century and used until today to diagnose disease [9]. Such acute and urgent care needs enabled the evolution of a centralized infrastructure system.

The model developed during the time when the scientific approach focused on the body as a machine and therefore disease to be the consequence of breakdown in the machine [10]. Therefore, the model is disease-oriented and reductionist, focusing on the identification of physical causes assuming that illness and symptoms arise from an underlying pathophysiology of cellular abnormalities or imbalances in homeostasis $[8,11]$. Such a model focus was very useful in addressing the pressing medical problems of the 19th and early 20th centuries, namely, infectious diseases and traumatic illness [12].

In contrast, the current healthcare system is facing an unprecedented chronic disease burden. These conditions, unlike acute conditions, are particularly complex in that they are ongoing and tend to involve multiple factors with multiple interactions between them [13]. Furthermore, they currently represent the leading causes of death and disability in the United States and globally $[14,15]$. As of 2012, 50\% of all adults had one or more chronic health conditions [16]. In the first time in history, our children's generation is expected to lead shorter life spans than our own [17]. Chronic diseases are also significantly increasing demand for healthcare services and driving up costs. As of $2010,86 \%$ of all healthcare spending was for people with one or more chronic medical conditions [18]. They account for $81 \%$ of hospital admissions; $91 \%$ of all prescriptions filled; and $76 \%$ of all physician visits [19].

Relative to acute conditions, the characteristics of chronic conditions present several new healthcare delivery challenges. Four are identified in Table 1.

To further distinguish between acute and chronic healthcare delivery, this work refers to "individuals" rather than patients. The former addresses people throughout their lives in general whereas the latter addresses their state when they are in a healthcare delivery facility.

Several definitions have been proposed for the term chronic disease [20]. All of which encompass a concept of either (1) unspecified long duration [21, 22] or (2) specified long duration lasting more than 12 months [23-27]. Defining chronic conditions with the key component of long duration emphasizes that the changes, in the individual's health state, occur over a period that is much longer than any single visit to a healthcare facility [20].

Goodman et al. demonstrate that most chronic disease definitions include a key component of need for ongoing medical care $[20,22-25,27,28]$. There is, however, an important property to such ongoing medical care, which is typically described by the term continuity, emphasizing that the sequence of events logically depend on each other. Haggerty et al. define continuity as "the degree to which a series of discrete healthcare events is experienced as coherent and connected and consistent with the individual's medical needs and personal context" [29]. Continuity has been shown to improve chronic disease outcomes [30,31].

Definitions of chronic diseases are generally very broad and describe key components rather than the specifics of a disease. This is primarily because the experience of a chronic condition may manifest uniquely for each individual based on several factors [32-36]. Our scientific models have been shifting from the classic biomedical model pervasive in acute care to the biopsychosocial model, which argues that the causes and consequences of illness exist at multiple levels of organization: biological, psychological, and social [10, 37]. Furthermore, the Institute of Medicine has identified individualcentered care as one of the six specific aims of improvement in healthcare and further emphasizes the importance of an individual's unique experience of a chronic condition [38]. This has led to the incorporation of shared decision-making [39] between healthcare providers and the individual. Allowing the individual to become a decision agent in their care significantly increases complexity with the need for cooperation, information sharing, and consensus in order to reach a decision which was classically dictated by the healthcare provider agent alone.

Finally, chronic conditions often affect many aspects of an individual's health that are often covered by disparate medical disciplines. This has led to the need for multiple specialties in treating a person with a chronic condition [40-44]. Requiring a much larger team of healthcare providers including specialists and non-MD clinicians has fundamentally changed the role (i.e., tasks) of the agent (i.e., healthcare provider) and their position in taking care of the patient. Furthermore, several healthcare delivery models (e.g., Collaborative Care Model [45] and Integrated Care Model [46]) have restructured to coordinate and/or co-locate care. Such embedded care models are effective systems within other systems that are likely to co-evolve. Systems embedded within 
TABLE 2: New healthcare delivery system requirements.

(1) Continues to deliver care well after the individual has left the healthcare facility.

(2) Deeply understands the health state of the individual.

(3) Manages individualized health outcomes.

(4) Coordinates numerous practitioners representing many medical specialties.

other systems may appear as healthcare delivery models within the same clinic as described or may be due to the changes from fee-for-service to value-based care [47] which have effectively widened the boundary of a system.

Thus, the characteristics of chronic diseases require much more from the operation of a healthcare delivery system than the way it has operated to address acute conditions within individual visits. Instead, the four characteristics presented in Table 1 present four new requirements on the healthcare delivery system as shown in Table 2 .

The current healthcare delivery system, designed from the outset to address acute conditions, is ill-suited to address the four requirements stated above. Furthermore, their fulfillment fundamentally changes, not just the relationships between the individual and the healthcare delivery system, but also the relationships between its many services and resources as well. Addressing and architecting the relationships between services and resources and those between the individual and the healthcare delivery system are critical to managing the complexity arising from these relationships since "the interactions within a complex adaptive system are often more important than the discrete actions of the individual parts" [48]. These relationships suggest the need for architecting a system model for personal healthcare delivery and managed individual health outcomes.

1.3. Mass-Customized Production Systems. While this strategic shift in healthcare delivery systems may appear dramatic, it is not without precedent in other domains. Mass production systems underwent a similar transformation to become mass-customized production systems $[49,50]$. In the 1990s, manufacturing became increasingly characterized by a continually evolving and an ever more competitive marketplace. The implementation of lean manufacturing principles had freed excess capacity and thus gave consumers greater influence over the quality, quantity, and variety of products $[51,52]$. In order to stay competitive, manufacturing firms had to respond with high variety products achieved through the use of flexible manufacturing systems and reconfigurable manufacturing systems $[49,50]$. Reconfigurable manufacturing systems, in particular, required a rearchitecting of production systems in favor of modular machine tools and distributed control systems in the form of multi-agent systems [53-64]. In time, these new architectural developments were situated within quantitative graph theoretic frameworks [6569] and used to design new mass-customized production systems $[70,71]$. This quantitative foundation now lends itself to reapplication for personalized healthcare delivery.
1.4. Paper Contribution. This paper architects a systems model for personal healthcare delivery and managed individual health outcomes. It serves to address the identified need for systems tool in medicine $[72,73]$. To support the development, it specifically roots itself in recent work on the architecture of mass-customized production systems and then incorporates features specific to healthcare delivery. This model directly addresses the four requirements derived from the characteristics of chronic diseases. Special attention will be given to the description of an individual's health state and its stochastic evolution in relation to the healthcare delivery system. This is in contrast to many existing works $[74,75]$, particularly in healthcare discrete-event simulation, where the individual is treated as a stateless passive entity (e.g., a Petrinet token) being pushed or pulled through various healthcare system queues.

The development of an architecture model opens several avenues for future work including cost-benefit analysis, discrete-event simulation, resilience analysis, optimization, and multi-agent systems.

1.5. Paper Outline. The paper first begins with the description of the architecture model (Section 2). Next, An Acute Care Illustrative Example (Section 3) and a Chronic Care Illustrative Example (Section 4) are presented and followed by Discussion (Section 5) and Conclusion (Section 6). The work assumes prerequisite knowledge in model-based systems engineering [76-79], graph theory [80, 81], and discreteevent simulation [82] which is otherwise gained from the cited texts.

\section{Development of Architecture Model}

The development of the architecture model proceeds in five parts following Figure 1. As found in many systems engineering texts $[76,77]$, the healthcare delivery system is characterized by its form, function, and concept. Section 2.1 describes the system form as a set of human and technical resources that make up a physical architecture. Section 2.2 describes the system function as a set of system processes that make up a functional architecture. Section 2.3 describes the system concept as an allocated architecture composed of a bipartite graph between the system processes and resources. Section 2.4 then introduces a discrete-event Petri-net model describing the evolution of an individual's health state. Here, the individual represents the primary value-adding operand of the healthcare delivery system. Its introduction addresses the first three requirements identified in the introduction. Finally, Section 2.5 introduces a bipartite graph that links the healthcare system function to the evolution of an individual's health state. To support the discussion, the architecture is presented graphically in SysML as well as quantitatively. The quantitative discussion draws heavily on analogous works on masscustomized production systems [65-71] and may be viewed as an extension of recent work on healthcare human resources management [83].

2.1. Healthcare System Form: Systems Resources and Their Flexible Aggregation. To begin, the healthcare system form 


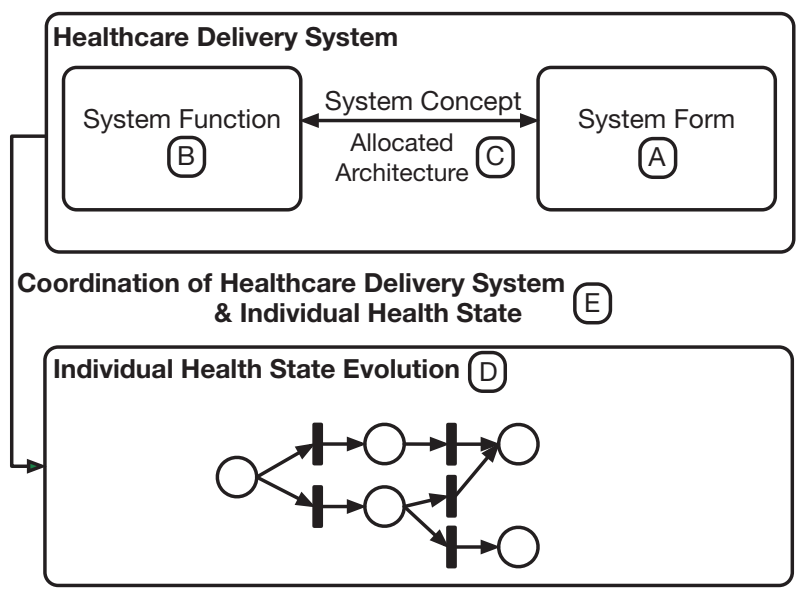

FIgURE 1: Healthcare System Architecture includes the healthcare delivery system, the individual health state, and their coordination.

$[76,77]$ (shown as A in Figure 1) is composed of several types of system resources which may be flexibly aggregated. In mass-customized production systems, the system resources were classified as transformation resources (i.e., value-adding machines), storage resources (i.e., independent buffers), and transportation resources (i.e., material handlers) [6571]. Each of these has their analogous counterparts in the healthcare delivery system. That said, the healthcare delivery system has several essential characteristics which require a finer classification of its system resources. These include the definition of measurement and decision resources as well as the distinction between human and technical resources. The remainder of the discussion on healthcare system form is guided by the SysML block diagram in Figure 2.

Definition 1 (transformation resource). A resource $\mathbb{r}_{F} \in \mathbb{R}_{F}$ capable of a transformative effect on its operand (e.g., the health state of an individual). They include human transformation resources $r_{F} \in R_{F}$ (e.g., surgeon, cardiologist, and psychologist) and technical transformation resources $r_{F} \in$ $\mathscr{R}_{F}$ (e.g., operating theatres, chemotherapy infusion room, and delivery room). Transformation resources are the set union of human and technical transformation resources, $\mathbb{R}_{F}=R_{F} \cup \mathscr{R}_{F}$.

Definition 2 (decision resource). A resource $\mathbb{r}_{D} \in \mathbb{R}_{D}$ capable of advising the operand, an individual, on how to proceed next with the healthcare delivery system. They include human decision resources $r_{D} \in R_{D}$ (e.g., oncologist, general practitioner, and surgeon) and technical decision resources $r_{D} \in$ $\mathscr{R}_{D}$ (e.g., decision support systems and electronic medical record decision tools). Decision resources are the set union of human and technical decision resources, $\mathbb{R}_{D}=R_{D} \cup \mathscr{R}_{D}$.

Decision resources are analogous to storage resources in previous work on mass-customized production systems [65-71] but are different in two regards. First and fundamentally, in production systems, a shop-floor controller (be it automatic or manual) often dispatches a passive product.
Naturally, within the medical community, an individual is viewed as an active stakeholder-participant within the healthcare delivery system rather than a passive entity. In this regard, recent work on "intelligent products" [84-86] in masscustomized production systems is a much more appropriate analogy. Such "intelligent products" are cyber-physical entities that consist of a physical product tied 1-to-1 with an informatic agent that is capable of negotiating and coordinating with the production system. Second, in production systems, intelligent products do not need to be in a specific location to be part of decisions for the next steps of production. In contrast, individuals must often meet healthcare professionals face-to-face in order to determine next steps. Consequently, the analogy to production system storage resources is retained because these decisions must occur at well specified locations in the healthcare delivery system.

Definition 3 (measurement resource). A resource $\mathbb{r}_{M} \in \mathbb{R}_{M}$ capable of measuring the operand: here the health state of an individual. They include human measurement resources $r_{M} \epsilon$ $R_{M}$ (e.g., MRI technician, sonographer, and phlebotomist) and technical measurement resources $r_{M} \in \mathscr{R}_{M}$ (e.g., magnetic resonance imaging scanner, ultrasound machine, and Holter monitor). Measurement resources are the set union of human and technical measurement resources, $\mathbb{R}_{M}=$ $R_{M} \cup \mathscr{R}_{M}$.

Measurement resources are analogous to storage resources in previous work on mass-customized production systems [65-71]. Fundamentally speaking, in production systems, a product's state is relatively well-known from the course of its production. Storage resources are required to simply account for a product's location. In contrast, an individual's health state needs to be explicitly ascertained by the healthcare delivery system. The analogy to production system storage resources is retained because naturally this measurement must occur at well specified locations in the healthcare delivery system.

Definition 4 (transportation resource). A resource $\mathbb{r}_{N} \in \mathbb{R}_{N}$ capable of transporting its operand: the individual themselves. They include human transportation resources $r_{N} \in R_{N}$ (e.g., emergency medical technician, clinical care coordinator, and surgical team member) and technical transportation resources $\mu_{N} \in \mathscr{R}_{N}$ (e.g., ambulance, gurney, and wheelchair). Transportation resources are the set union of human and technical transportation resources, $\mathbb{R}_{N}=R_{N} \cup \mathscr{R}_{N}$.

Transportation resources act much like they do in masscustomized production systems. However, healthcare transportation resources are only required when the individual is no longer able to transport themselves unassisted within the healthcare delivery system.

Definition 5 (buffer resource). A resource $r \in \mathbb{R}_{B}$ where

$$
\mathbb{R}_{B}=\mathbb{R}_{F} \cup \mathbb{R}_{D} \cup \mathbb{R}_{M} .
$$

In order to support the discussion of transportation processes, it is useful to introduce the concept of buffer 


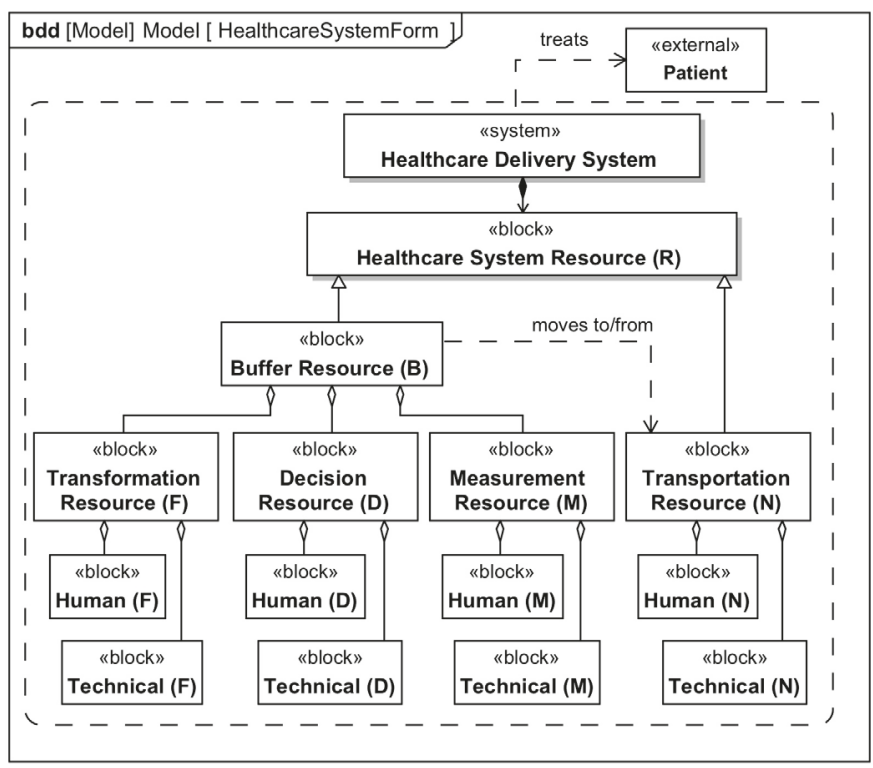

FIGURE 2: SysML block diagram of healthcare system form.

resources shown in Figure 2 and Definition 5. Collectively, they denote specified locations. In production systems, they were the set union of transformation and storage resources. Here, they are the set union of transformation, measurement, and decisions resources.

Furthermore, it is often useful to view healthcare delivery system resources purely in terms of human and technical classifications.

Definition 6 (human resource). A resource $r \in R$ where

$$
R=R_{F} \cup R_{D} \cup R_{M} \cup R_{N}
$$

Definition 7 (technical resource). A resource $r \in \mathscr{R}$ where

$$
\mathscr{R}=\mathscr{R}_{\mathscr{F}} \cup \mathscr{R}_{\mathscr{D}} \cup \mathscr{R}_{\mathscr{M}} \cup \mathscr{R}_{\mathcal{N}} .
$$

The healthcare delivery system resources described thus far allows specific instances to be non-uniquely classified. In the cases where a specific resource is capable of performing several processes, it must be uniquely classified. For example, a surgeon is trained and defined by their transformation ability and not just their decision capability. In order to create a unique classification of these resources, a set of ordered classification rules are implemented.

Definition 8 (rules for classification of healthcare system resources).

Rule 1. If $r \in R$ can Transform; then $r \in R_{F}$. If $r \in \mathscr{R}$ can Transform; then $r \in \mathscr{R}_{\mathscr{F}}$.

Rule 2. If $r \in R$ can Decide; then $r \in R_{D}$. If $r \in \mathscr{R}$ can Decide; then $r \in \mathscr{R}_{\mathscr{D}}$.
Rule 3. If $r \in R$ can Measure; then $r \in R_{M}$. If $r \in \mathscr{R}$ can Measure; then $r \in \mathscr{R}_{\mathscr{M}}$.

Rule 4. Otherwise $r \in R_{N}$ and $r \in \mathscr{R}_{\mathscr{N}}$.

These rules effectively sort resources on the basis of their most valuable capabilities. It is assumed that, with respect to value, Transform $>$ Decide $>$ Measure $>$ Transportation. This prioritization is based on healthcare resource hierarchical medical value to the healthcare system. In healthcare delivery systems, the value for these different capabilities has pushed the system to encourage clinicians to "practice at the top of their license."

As many healthcare systems have hundreds or thousands of personnel and equipment, it is useful to form aggregated resources $\overline{\mathbb{R}}[65-67,70,83]$.

$$
\overline{\mathbb{R}}=A_{R} \circledast \mathbb{R},
$$

where $\circledast$ is an aggregation operator and $A_{R}$ is an aggregation matrix $[65-67,70,83]$. These aggregations are flexible and logical in nature and can be changed administratively. For example, an Orthopedic Care Team may be composed of a surgeon, an anesthesiologist, nurses, surgical techs, residents/ medical students, and cleaning staff. Naturally, the composition of this aggregation can be changed at a later time. Healthcare resource aggregation is critical for allowing flexibility in the level of abstraction (i.e., individual, teams, departments, clinics, and regions or state) of the system.

In summary, healthcare delivery system resources are the set union of these previously mentioned types of resources.

$$
\begin{aligned}
\mathbb{R} & =R_{F} \cup \mathscr{R}_{F} \cup R_{D} \cup \mathscr{R}_{D} \cup R_{M} \cup \mathscr{R}_{M} \cup R_{N} \cup \mathscr{R}_{N}, \\
\mathbb{R} & =\mathbb{R}_{F} \cup \mathbb{R}_{D} \cup \mathbb{R}_{M} \cup \mathbb{R}_{N}, \\
\mathbb{R} & =R \cup \mathscr{R} .
\end{aligned}
$$


2.2. Healthcare System Function. Healthcare system function $[76,77]$ (shown as B in Figure 1) is composed of several types of system processes which will ultimately be deployed by the system resources. In mass-customized production systems, the system processes were classified as two types: transformation and transportation [65-71]. Storage processes were considered as transportation processes with nondistinct origin and destination [65-71]. Here, the focus was on physical processes that directly interacted with the value-adding operand, the mass-customized product. Analogously, transformation and transportation processes exist similarly in the healthcare delivery system as physical processes on the individual. That said, the healthcare delivery system has several essential characteristics that requires a broader classification. The engineering systems literature often classifies processes into five: transformation, transportation, storage, control, and exchange [87]. Consequently, in healthcare, measurement processes are identified as a type of control process and collaborative decisions are identified as a type of exchange process. It is important to note that these are cyber-physical processes in that they require the physical presence of the valueadding operand (i.e., the individual) as well as information flow between the individual and the healthcare delivery system (and its resources). This classification scheme is summarized by the SysML block diagram in Figure 3 .

As with mass-customized production systems [65-71], these system processes may be organized to make up a (generic) template model of healthcare delivery system function. These functions are based on a diagnostic model [88] that first examines the patient's complaint (measure), second, attempts to determine its cause (diagnose and decide) and, third, applies a treatment regimen to that cause (treat or transform). Sequentially, these are

(1) measurement: understand, quantify or classify individual state,

(2) decision: determine what to do for the individual and when,

(3) transformation: perform service(s) for the individual,

(4) transportation: move the individual between any of these processes.

Figure 4 shows this template service model graphically. Each of these is now described in detail.

Definition 9 (transformation process). A physical process $p_{F} \in P_{F}$ that transforms the operand: specifically the internal health state of the individual (i.e., treatment of condition, disease, or disorder).

A transformation process typically changes the internal health state of the individual. Such processes include surgical procedures (e.g., amputation, ablation, laparoscopic surgery, and endoscopic surgery) and therapeutic procedures (e.g., pharmacotherapy, chemotherapy, physical therapy, psychotherapy, and laser therapy).

Definition 10 (decision process). A cyber-physical process $p_{D} \in P_{D}$ occurring between a healthcare system resource and the operand: the individual that generates a decision on how to proceed next with the healthcare delivery system.

Several types of decision processes exist in a healthcare delivery system. Planning is defined as the determination of which healthcare system processes need to occur for the individual (e.g., treatment plan and cancer screening plan). Scheduling is defined as who/what is going to perform that process and when (e.g., individual booking). Furthermore, it is important to distinguish between intermediate and dispatching decisions where the latter serve to trigger physical activities to the individual and the former do not.

As a physical process, the individual must be physically present at a healthcare system resource (buffer) and in that sense a decision process resembles a storage process. As an informatic (i.e., cyber) process, information is exchanged (in both directions) between the individual and the healthcare system resource to support collaborative decision-making [39]. A critical aspect of shared decision-making and information exchange includes the healthcare system resource educating the individual. This enhances the individual's ability to make the most beneficial medical and behavioral decisions. If the individual is incapacitated, then the healthcare system resource makes the decision autonomously.

Definition 11 (measurement process). A cyber-physical process $p_{M} \in P_{M}$ that converts a physical property of the operand into a cyber, informatic property to ascertain health state of the individual.

Typical healthcare measurement processes acting on individuals include clinical evaluation, diagnostic tests (e.g., blood test, urine test, and stool test) and diagnostic procedures (e.g., medical imaging, endoscopy, and electrocardiography).

As a physical process, the individual must be physically present at a healthcare system resource (buffer) and in that sense a measurement process resembles a storage process. As an informatic (i.e., cyber) process, information is drawn from the individual to ascertain their health state (i.e., diagnose). In mass-customized production systems, the state of each product is relatively well-known from the course of its production. In contrast, an individual's health state evolves stochastically and spontaneously. Understanding an individual's health state is one of the core functions or processes of the healthcare system, which it performs through measurement.

Definition 12 (transportation process). A physical process $p_{N} \in P_{N}$ that moves individuals between healthcare resources (e.g., bring individual to emergency department and move individual from operating to recovery room).

Although individuals do not typically need to be moved (unless incapacitated), transportation processes are specifically included for the sake of completeness and adherence to the mass-customized production system analogy. This is also performed because it explicitly states the capabilities of the system rather than the utilization of the system by the operand. 


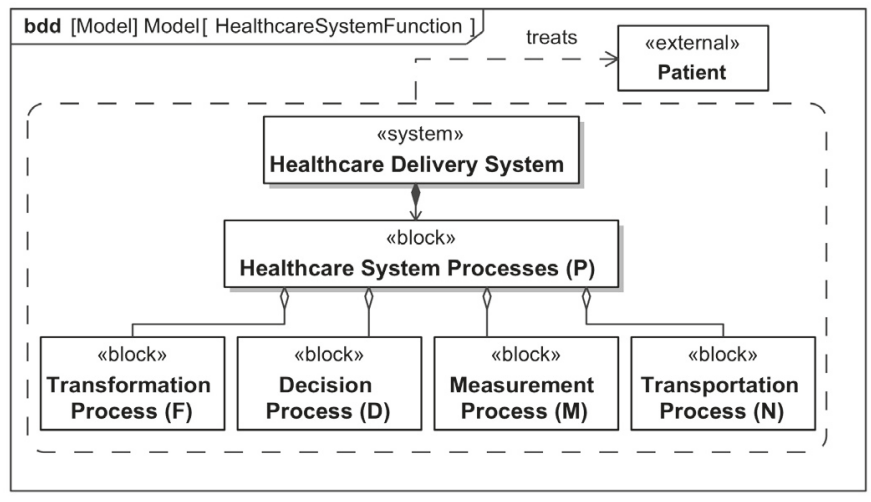

FIGURE 3: SysML block diagram of healthcare system function.

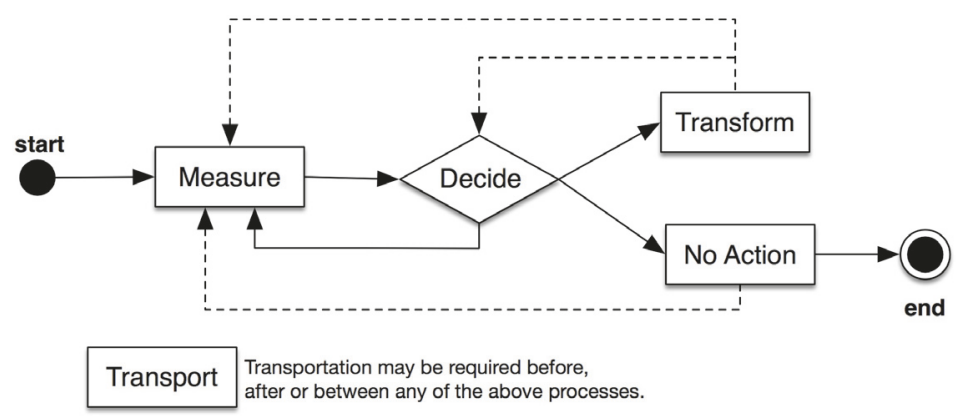

FIGURE 4: Healthcare functional model concept of healthcare system processes. Solid lines represent typical process sequences and dotted lines represent possible process sequences.

Furthermore, the introduction of the set of buffer resources $\mathbb{R}_{B}$ implies that there are $\sigma^{2}\left(\mathbb{R}_{B}\right)$ transportation processes, where the $\sigma()$ notation is introduced to give the size of a set. As a matter of convention, a healthcare process $p_{N u}$ transports an individual from resource $r_{y_{1}} \in \mathbb{R}_{B}$ to resource $r_{y_{2}} \in \mathbb{R}_{B}$ such that [65-71]

$$
u=\sigma\left(\mathbb{R}_{B}\right)\left(y_{1}-1\right)+y_{2} .
$$

Definition 13 (non-transportation process). A combination of non-transportation processes representing transformation, decision, and measurement process, $p_{B} \in P_{B}$, is a set union of non-transportation processes.

$$
P_{B}=P_{F} \cup P_{D} \cup P_{M}
$$

As many healthcare systems have hundreds or thousands of processes, it is often useful to form aggregated processes $\bar{P}$.

$$
\bar{P}=A_{P} \circledast P,
$$

where $\circledast$ is an aggregation operator and $A_{P}$ is an aggregation matrix. These aggregations are flexible and logical in nature. Since the healthcare sector has been so heavily influenced by fee-for-service reimbursement strategies, there have been many efforts to codify many of these services or processes to various degrees in various specialties.
In summary, the healthcare system processes are the set union of transformation, decision, measurement, and transportation processes.

$$
P=P_{F} \cup P_{D} \cup P_{M} \cup P_{N} .
$$

2.3. Healthcare System Concept (Knowledge Base). Now that healthcare system function and form have been described, the allocation of their constituent processes to their associated resources can be presented. System concept is defined as an allocated architecture composed of a bipartite graph between the system processes and resources (shown as $\mathrm{C}$ in Figure 1). This is an integral aspect of many common engineering design methodologies [76, 89-91]. Here, this work builds upon Axiomatic Design Theory, and more specifically for Large Flexible Engineering Systems [68, 92] where this allocation is mathematically formalized in terms of a "design equation" [65-71].

$$
P=J_{S} \odot \mathbb{R},
$$

where $J_{S}$ is a binary matrix called a "system knowledge base" and $\odot$ is "matrix Boolean multiplication" [65-71].

Definition 14 (system knowledge base [65-71]). A binary matrix $J_{S}$ of size $\sigma(P) \times \sigma(\mathbb{R})$ whose element $J_{S}(w, v) \in\{0,1\}$ is equal to one when event $e_{w v} \in \mathscr{E}_{\delta}$ (in the discrete-event systems sense [82]) exists as a system process $p_{w} \in P$ being executed by a resource $r_{v} \in \mathbb{R}$. 
This system knowledge base definition has been applied to mass-customized production systems [65-71], transportation systems [93-95], water systems [68, 96, 97], and electric power systems [98] and is likely suitable to the healthcare delivery system as another instance of the class of Large Flexible Engineering Systems. It emphasizes the elemental capabilities that exist within the system.

It is important to note that the healthcare delivery system knowledge base $J_{S}$ has a special structure that can be determined from smaller knowledge bases that individually address transformation, decision, measurement, and transportation processes. Using the rules presented in Definition 8, it follows that

$$
\begin{aligned}
& P_{F}=J_{F} \odot \mathbb{R}_{F}, \\
& P_{D}=\left[\begin{array}{ll}
J_{F D} & J_{D}
\end{array}\right] \odot\left(\mathbb{R}_{F} \cup \mathbb{R}_{D}\right), \\
& P_{M}=\left[\begin{array}{lll}
J_{F M} & J_{D M} & J_{M}
\end{array}\right] \odot\left(\mathbb{R}_{F} \cup \mathbb{R}_{D} \cup \mathbb{R}_{M}\right) \text {, } \\
& P_{N}=\left[\begin{array}{llll}
J_{F N} & J_{D N} & J_{M N} & J_{N}
\end{array}\right] \\
& \odot\left(\mathbb{R}_{F} \cup \mathbb{R}_{D} \cup \mathbb{R}_{M} \cup \mathbb{R}_{N}\right) \text {. }
\end{aligned}
$$

Consequently,

$$
J_{S}=\left[\begin{array}{cccc}
J_{F} & 0 & 0 & 0 \\
J_{F D} & J_{D} & 0 & 0 \\
J_{F M} & J_{D M} & J_{M} & 0 \\
J_{F N} & J_{D N} & J_{M N} & J_{N}
\end{array}\right] .
$$

The elemental capabilities that exist within the healthcare delivery system may not always be available. In the operational time frame, constraints may apply that effectively eliminate events from the event set. The existence of such constraints is captured within a system events constraints matrix.

Definition 15 (system events constraints matrix [65-71]). A binary matrix $K_{S}$ of size $\sigma(P) \times \sigma(\mathbb{R})$ whose element $K_{S}(w, v) \in\{0,1\}$ is equal to one when a constraint eliminates event $e_{w v}$ from the event set.

Such constraints can be applied on technical resources in the form of breakdowns or maintenance. Similarly, human resources may call in sick or request other types of time off.

The construction of $J_{S}$ and $K_{S}$ allows the enumeration of the healthcare system's structural degrees of freedom.

Definition 16 (structural degrees of freedom [65-71]). The set of independent actions $\psi_{i} \in \mathscr{E}_{\mathcal{S}}$ that completely define the available processes in the system. Their number is given by

$$
\begin{aligned}
\operatorname{DOF}_{S} & =\sigma\left(\mathscr{E}_{S}\right)=\sum_{w}^{\sigma(P)} \sum_{v}^{\sigma(\mathbb{R})}\left[J_{S} \ominus K_{S}\right](w, v) \\
& =\sum_{w}^{\sigma(P)} \sum_{v}^{\sigma(\mathbb{R})} A_{S}(w, v)
\end{aligned}
$$

where $\ominus$ is Boolean subtraction $(A \ominus B=A \cdot \bar{B}$, where $A \cdot B$ is the Hadamard product or equivalently matrix AND for Booleans. $\bar{B}=\mathrm{NOT}(B))$. These structural degrees of freedom enumerate the capabilities of the healthcare delivery system independent of their sequence. They have been shown to be an essential step in determining the system behavior of several Large Flexible Engineering Systems including mass-customized production systems [65-71], transportation systems [9395], water systems $[68,96,97]$, and electric power systems [98].

From an architectural perspective, the structural degrees of freedom serve to construct a heterofunctional network $[68,92]$ that describes the structure of the healthcare delivery system. Such a network describes feasible sequences of pairs of structural degrees of freedom called strings. Consider two arbitrary structural degrees of freedom $e_{w_{1} v_{1}}$ and $e_{w_{2} v_{2}}$. Their corresponding string is $z_{\psi_{1} \psi_{2}}=e_{w_{1} v_{1}} e_{w_{2} v_{2}} \in Z$ where $\psi_{1}=$ $\sigma(P)\left(w_{1}-1\right)+w_{2}$ and $\psi_{2}=\sigma(\mathbb{R})\left(v_{1}-1\right)+v_{2} \forall w_{1}, w_{2} \in$ $\{1, \sigma(P)\}$ and $\forall v_{1}, v_{2} \in\{1, \sigma(\mathbb{R})\}$. The existence of these strings can be captured in a system sequence knowledge base $J_{\rho}$.

Definition 17 (system sequence knowledge base [65-71]). A square binary matrix $J_{\rho}$ is of size $\sigma(P) \sigma(\mathbb{R}) \times \sigma(P) \sigma(\mathbb{R})$ whose element $J_{\rho}\left(\psi_{1}, \psi_{2}\right) \in\{0,1\}$ is equal to one when string $z_{\psi_{1} \psi_{2}}$ exists. It may be calculated directly as

$$
J_{\rho}=\left[J_{S} \cdot \bar{K}_{S}\right]^{V}\left[J_{S} \cdot \bar{K}_{S}\right]^{V T}
$$

where ()$^{V}$ is shorthand for vectorization (i.e., $\left.\operatorname{vec}()\right)$.

As before, there may exist sequence-dependent constraints that eliminate some of these two-event strings. These are captured within a system sequence constraints matrix.

Definition 18 (system sequence constraints matrix [65-71]). A square binary constraints matrix $K_{\rho}$ of size $\sigma(P) \sigma(\mathbb{R}) \times$ $\sigma(P) \sigma(\mathbb{R})$ whose elements $K\left(\psi_{1}, \psi_{2}\right) \in\{0,1\}$ are equal to one when string $z_{\psi_{1} \psi_{2}}=e_{w_{1} v_{1}} e_{w_{2} v_{2}} \in Z$ is eliminated.

Unlike $K_{S}$ where a zero matrix is possible, it has been shown in prior work [65-69] that the system sequence constraints matrix $K_{\rho}$ has perpetually binding constraints that arise from the functional architecture. In mass-customized production systems, these include, at a minimum, continuity relations that ensure the destination of the first structural degree of freedom is equivalent to the origin of the second [65-69]. Extensive discussions have been provided on the sources of additional sequence-dependent constraints [6567]. Healthcare delivery systems naturally observe the constraints from continuity relations. They also have many constraints arising from clinical medical practice and administration. Examples of these are discussed in greater detail in Section 5.

Finally, the construction of $J_{\rho}$ and $K_{\rho}$ allows the construction of an adjacency matrix $A_{\rho}$ that describes a heterofunctional network.

$$
A_{\rho}=J_{\rho} \ominus K_{\rho}
$$


Definition 19 (system sequence degrees of freedom [65-71]). The set of independent actions $\mathscr{E}_{\rho}$ that completely defines the available sequence processes in the system. Their number is given by

$$
\begin{aligned}
\operatorname{DOF}_{\rho} & =\sigma\left(\mathscr{E}_{\rho}\right)=\sum_{\psi_{1}}^{\sigma(P)} \sum_{\psi_{2}}^{\sigma(\mathbb{R})}\left[J_{\rho} \ominus K_{\rho}\right]\left(\psi_{1}, \psi_{2}\right) \\
& =\sum_{\psi_{1}}^{\sigma(P)} \sum_{\psi_{2}}^{\sigma(\mathbb{R})} A_{\rho}\left(\psi_{1}, \psi_{2}\right) .
\end{aligned}
$$

Here, the nodes represent structural degrees of freedom and the edges represent system sequence degrees of freedom as the feasible sequences between them. The adjacency matrix $A_{\rho}$ has been shown in prior work to affect the resilience properties of Large Flexible Engineering Systems including masscustomized production systems [65-71], transportation systems [93-95], water systems $[68,96,97]$, and electric power systems [98].

In summary, the healthcare system concept is captured in the system knowledge base $J_{S}$ and the system sequence knowledge base $J_{\rho}$ to describe the system's capabilities individually and in pairs. This also requires their corresponding constraint matrices $K_{S}$ and $K_{\rho}$. These capability and constraint matrices allow for the construction of a heterofunctional network adjacency matrix $A_{\rho}$ where the nodes represent the structural degrees of freedom $\mathscr{E}_{S}$ and the edges represent their feasibility as pairs.

2.4. Individual's "Clinical" Health State Evolution. With the architecture of the healthcare delivery system in place, the discussion turns to an individual's health state evolution (shown as D in Figure 1). While it is important to quantify the capabilities of the healthcare delivery system, it is equally critical to introduce the evolution of each individual's health state so as to keep track of individual patient outcomes. Ultimately, this is necessary to meet the requirements presented in Table 2 so as to address healthcare delivery challenges posed by chronic conditions described in Table 1 .

It is here that the analogy between a personalized healthcare delivery system and mass-customized production systems firmly takes shape. In mass-customized production systems, each product is assumed to be entirely different from the one before it. For example, Mercedes Benz offered $3.347807348 \times 10^{24}$ variations on their Mercedes E class model in 2002 [99]. Human individuals are also unique. From the healthcare delivery system's perspective, the International Classification of Diseases (ICD), currently at ICD-10, has 68,000 diagnosis codes [100]. When one considers that $25 \%$ of Americans have multiple chronic conditions [26], the number of possible combinations is essentially equal to the population. In both cases, there exist a large number of unique operands that utilize different capabilities of their respective systems. Therefore, a systematic approach is required to model each individual.

In terms of modeling each individual, one must distinguish between the bio-physical-chemical continuous health state of the individual, often found in systems biology [101, 102] and an individual's clinical health state. The clinical health state is often ascertained by the clinician through differential diagnosis $[103,104]$. The process of diagnosis generally includes a form of discrete classification such as by type (e.g., Type 1 diabetes versus Type 2 diabetes [105]), stage (e.g., Breast Cancer Stage IA versus Stage IIIC [106]), grade (e.g., Brain Tumor Grade II diffuse astrocytoma versus Grade IV glioblastoma [107]) or class (e.g., Heart Failure Functional Class I versus Heart Failure Functional Class IV [108]). Furthermore, the evolution of that state happens at irregular time intervals and often as a result of specific events be they from the healthcare delivery system (e.g., surgery), the environment (e.g., exposure to allergens), or new behavior (e.g., a new exercise regimen). Therefore, it is more appropriate to use a discrete-event system model to describe the evolution of an individual's clinical health state.

To continue the analogy, in mass-customized production systems, the evolution of a product's state from raw good to finished product was described by a deterministic untimed Petri-net called a "Product Net" [67]. Similarly, a "Health Net" is introduced, this time as a fuzzy timed Petri-net, to model an individual's clinical health state.

Definition 20 (Health Net). Given an individual $l_{i}$, that is part of a population $L$, where $L=\left\{l_{1}, \ldots, l_{\sigma(L)}\right\}$, the evolution of their clinical health state can be described as a fuzzy timed Petri-net [109-111]:

$$
N_{l_{i}}=\left\{S_{l_{i}}, \mathscr{E}_{l_{i}}, \mathscr{M}_{l_{i}}, W_{l_{i}}, D_{l_{i}}, Q_{l_{i}}\right\}
$$

where

(i) $N_{l_{i}}$ is the Health Net;

(ii) $S_{l_{i}}$ is the set of places describing a set of health states;

(iii) $\mathscr{E}_{l_{i}}$ is the set of transitions describing health events;

(iv) $\mathscr{M}_{l_{i}} \subseteq\left(S_{l_{i}} \times \mathscr{E}_{l_{i}}\right) \cup\left(\mathscr{E}_{l_{i}} \times S_{l_{i}}\right)$ is the set of arcs describing the relations of health states to health events or health events to health states;

(v) $W_{l_{i}}$ is the set of weights on the arcs describing the health transition probabilities for the arcs;

(vi) $D_{l_{i}}$ is the set of transition durations;

(vii) $Q_{l_{i}}$ is the Petri-net marking representing the likely presence of the set of health states as a discrete probabilistic state.

The Petri-net structure leads directly to the definition of its discrete-event dynamics.

Definition 21 (fuzzy timed Petri-net (discrete-event) dynamics [112]). Given a binary input firing vector $U_{l_{i}}^{+}[k]$ and a binary output firing vector $U_{l_{i}}^{-}[k]$, both of size $\sigma\left(\mathscr{E}_{l_{i}}\right) \times 1$, and the positive and negative components $\mathscr{M}_{l_{i}}^{+}$and $\mathscr{M}_{l_{i}}^{-}$of the Petri-net incidence matrix of size $\sigma\left(S_{l_{i}}\right) \times \sigma\left(\mathscr{E}_{l_{i}}\right)$, the evolution of the marking vector $Q_{l_{i}}$ is given by the state transition function $\Phi\left(Q_{l_{i}}[k], U_{l_{i}}[k]\right)$ :

$$
Q_{l_{i}}[k+1]=\Phi\left(Q_{l_{i}}[k], U_{l_{i}}^{-}[k], U_{l_{i}}^{+}[k]\right),
$$

where $Q_{l_{i}}=\left[Q_{S_{l_{i}}} ; Q_{\mathscr{E}_{l_{i}}}\right]$ and 


$$
\begin{aligned}
& Q_{S_{l_{i}}}[k+1]=Q_{S_{l_{i}}}[k]+\mathscr{M}_{l_{i}}^{+} U_{l_{i}}^{+}[k]-\mathscr{M}_{l_{i}}^{-} U_{l_{i}}^{-}[k], \\
& Q_{\mathscr{C}_{l_{i}}}[k+1]=Q_{\mathscr{C}_{l_{i}}}[k]-U_{l_{i}}^{+}[k]+U_{l_{i}}^{-}[k] .
\end{aligned}
$$

$Q_{S_{l_{i}}}$ is introduced to probabilistically mark Petri-net places whereas $Q_{\mathscr{C}_{i}}$ is introduced to mark the likelihood that a timed transition is currently firing. The transitions are fired based on a scheduled event list that combines the discrete events with a time interval.

Definition 22 (scheduled event list [82]). A tuple $\mathcal{S}_{l_{i}}=$ $\left(u_{l_{i} \psi}[k], t_{k_{l_{i}}}\right)$ consists of all elements $u_{l_{i} \psi}[k]$ in firing vectors $U_{l_{i}}^{-}[k]$ and their associated times $t_{k_{l_{i}}}$. For every element, $u_{l_{i}^{-}}[k] \in U_{l_{i}}^{-}[k]$, there exists another element $u_{l_{i \psi}^{+}}[\kappa] \in U_{l_{i}}^{+}[\kappa]$ which occurs at time $t_{\kappa_{l_{i}}}, d_{\psi_{l_{i}}}$ time units later. $t_{\kappa_{l_{i}}}=t_{k_{l_{i}}}+d_{\psi_{l_{i}}}$.

The Health Net is a practical representation of an individual's health state evolution from a clinical practitioner's perspective. Health states may include specific health factors (e.g., BMI level and glucose level) or may represent specific outcomes (e.g., pain level and cancer remission). The health events allow for the progression from one health state to the next as has been described in the scientific medical literature. The weights $W_{l_{i}}$ on the arcs $\mathscr{M}_{l_{i}}$ are no longer integers but instead probabilities of (1) a health state leading to a health event or (2) a health event leading a health state. The introduction of event timing and fuzzy state evolution are now specifically included to account for the requirements presented in Table 2.

An individual's health events $\mathscr{E}_{l_{i}}$ may be further classified. $\mathscr{E}_{l_{i}}=\mathscr{E}_{F l_{i}} \cup \mathscr{E}_{\varphi l_{i}}$. Each health event in $\mathscr{E}_{F l_{i}}$ is triggered by the transformation processes $P_{F}$ in the healthcare delivery system. Each health event in $\mathscr{E}_{\varphi l_{i}}$ is the result of a stochastic human process $P_{\varphi}$. These stochastic human processes (i.e., the capability of the human body to change health state without a healthcare delivery system trigger) may occur randomly for unknown reasons or it may be mediated by non-healthcare delivery system factors that may be internal or external to the individual, such as injury and social, economic, environmental, or biologic/genetic factors (e.g., car accident, BMI, and gender). Note that in mass-customized productions systems $\mathscr{E}_{\varphi l_{i}}$ do not exist. Furthermore, while the mass-customized production system describes a production transformation process (e.g., milling and painting) as having a single deterministic outcome, the scientific medical literature describes healthcare transformation processes (e.g., cancer therapy) as having several probabilistic health outcomes (e.g., cancer recurrent or cancer in remission) which would be reflected in the partially marked state $Q_{l_{i}}$.

Finally, in mass-customized production systems, the product net had events that occurred instantaneously. In contrast, the Health Net has events with stochastic duration. This is particularly important as an individual's health recovers and degrades at different rates.

With the Health Net model in place, it becomes important to understand how the full evolution of the clinical health states can be partitioned into episodes.
Definition 23 (episode). A partition of the Health Net $N_{e l_{i}}=$ $\left\{S_{e l_{i}}, \mathscr{E}_{e l_{i}}, \mathscr{M}_{e l_{i}}, W_{e l_{i}}, Q_{e l_{i}}\right\} \subset N_{l_{i}}$ describing a single noteworthy happening characterized by an underlying condition be it acute or chronic.

The set of episodes are assumed to be collectively exhaustive of the Health Net. $N_{l_{i}}=\bigcup_{j} N_{e_{j} l_{i}}$. Furthermore, with respect to health events, episodes are mutually exclusive; $\bigcap_{j} \mathscr{E}_{e_{j} l_{i}}=\varnothing$.

The definition of health nets and episodes allows a return to the central premise of the paper summarized in Tables 1 and 2. More specifically, episodes can be classified as either Acute or Chronic.

Definition 24 (acute condition). Acute condition occurs as an episode (e.g., infection, trauma, and fracture) with a short clinical course that usually responds to treatment where a return to a state of complete-pre-morbid health is the rule [113].

This definition facilitates two assumptions. (1) Acute conditions are mutually exclusive. $\bigcap_{j} N_{a_{j} l_{i}}=\varnothing$, which implies that for $n$ acute conditions

$$
\mathscr{M}_{l_{i}}=\left[\begin{array}{ccc}
\mathscr{M}_{a_{1} l_{i}} & 0 & 0 \\
0 & \ddots & 0 \\
0 & 0 & \mathscr{M}_{a_{n} l_{i}}
\end{array}\right] .
$$

(2) The duration of an acute episode occurs on the order of duration of a facility visit. This explains why the primary focus of many works on discrete-event simulation in the healthcare delivery system literature $[74,75]$ is on minimizing transportation and wait times.

Definition 25 (chronic condition). Chronic conditions occur as episodes (e.g., diabetes mellitus, arthritis, cardiovascular disease, and cancer) that have a protracted, usually more than 6 months clinical course (in many cases lifelong), requiring long-term therapy where response is suboptimal and return to a state of complete or pre-morbid normalcy is the exception [113].

Consequently, and unlike acute conditions, chronic conditions are not mutually exclusive. $\bigcap_{j} N_{c_{j} l_{i}}=\varnothing$. Furthermore, the duration of a chronic episode is much longer than duration of a facility visit, and therefore health events may occur both inside and outside the clinic.

In summary, it is important to recognize that the Health Net fulfills three of the requirements in Table 2. It specifically understands the clinical health state of an individual. It also tracks this health state as individuals reach favorable health outcomes. Finally, it recognizes that health events can be part of chronic episodes that are of long duration that can occur well after the individual has left the healthcare facility.

2.5. Linking Healthcare System State with Individual Health State. In order to link the transformation processes of the Healthcare Delivery System to the Individual Clinical Health 
State Evolution, a coordination link is necessary (shown as E in Figure 1).

In mass-customized production systems the linking between the production system state and the product state is captured using the product transformational feasibility matrix [65-71], where each transformation process in the production system induces a product event. Analogously, each transformation process in the healthcare delivery system induces its corresponding health event. For each individual, $l_{i}$, this feasibility condition can be captured in a binary individual transformational feasibility matrix.

Definition 26 (individual transformation feasibility matrix $\Lambda_{F_{i}}$ [65-71]). A binary matrix of size $\sigma\left(\mathscr{E}_{l_{i}}\right) \times \sigma\left(P_{F}\right)$, where $\Lambda_{F_{i}}(x, j)=1$ if transformational process $p_{F_{j}}$ realizes the health event $e_{x l_{i}}$.

Since each transformation process realizes exactly one individual health event, the sum of each column of the individual transformational feasibility matrix must equal one. The sum of each row gives the number of times that each transformation process is required by the individual.

Note that in mass-customized production systems, there are typically more unique transformation processes than in all the mass-customized products being produced [114]. In contrast, the healthcare delivery system typically only has transformation processes if they serve to improve individuals' health state. Meanwhile, all the health events in $\mathscr{E}_{\varphi l_{i}} \forall l_{i}$ are entirely autonomous of the healthcare delivery system.

2.6. Architecture Model Summary. This section has presented a personalized healthcare delivery system model following the conceptual depiction in Figure 1. The analogy between mass-customized production systems and personalized healthcare delivery is summarized in Table 3 and follows the nomenclature of the conceptual depiction in Figure 1.

\section{Acute Care Illustrative Example}

Now that the system architecture model has been developed in detail in Section II, it is used to model an acute episode of an ACL injury and repair as an illustrative example. Section 3.1 provides a narrative of an acute episode composed of several health events. Sections 3.2 and 3.3 parse this narrative into quantitative models of the Healthcare Delivery System and the Individual's Health State Evolution, respectively.

3.1. Description of Orthopedic Case. A typical example orthopedic case study of an ACL injury and repair is described below, drawn from a textbook clinical case [115].

Case Study 1. "Adam injured his left knee playing rugby when he fell forwards and sideways while the left foot remained fixed on the ground. He felt immediate pain and was unable to continue with the game. Pain and swelling increased over the next 2 hours. He was seen in an emergency department (ED) and $\mathrm{X}$-rays were negative for fractures. He was prescribed anti-inflammatories, given elbow crutches and advice on ice, rest and elevation. A clinic appointment to see an orthopedic consultant was arranged.

The orthopedic clinician (Ortho) evaluated the individual through a battery of special tests: anterior drawer test and valgus stress instability and active Lachman's test all of which were not conclusive due to pain and swelling. The individual received an urgent MRI scan which showed a rupture of the left ACL and a medial collateral ligament tear. Surgery was performed followed by an ACL post-operative rehabilitation protocol at physical therapy $(\mathrm{PT}) . "$

\subsection{Modeling the Healthcare Delivery System of the Orthopedic} Case. To begin the modeling of the Healthcare Delivery System, system Form and Function were determined by identifying the resources and processes mentioned in the text of Case Study 1. These were used to construct the system knowledge base $J_{S}$ as shown in Figure 5.

At this low-level of abstraction, the resources and processes do not reflect the typical practice of clinical operation and are instead aggregated to a higher level using equation (4). The term "orthopedic surgery" now describes an aggregated resource composed of human and technical resources of the orthopedic surgery team, room, and equipment. A similar aggregation is performed on the processes using equation (10) to aggregate the decision and decision support processes. Finally, a careful inspection of $J_{S}$ in Figure 5 shows that all resources are connected via transportation degrees of freedom. If these transportation capabilities are assumed to be always available, of relatively short duration and of sufficient capacity, then they can be eliminated without loss of generality from the knowledge base so as to focus on the more valuable healthcare delivery capabilities of transformation, decision, and measurement. These steps yield the knowledge base $\bar{J}_{F D M}$ at a higher level of aggregation as shown in Figure 6. For simplicity, the system is assumed to not have any event constraints during this acute episode. $K_{S}=0$. The associated number of structural degrees of freedom is calculated from equation (18). $\mathrm{DOF}_{S}=14$.

Continuing with the knowledge base $\bar{J}_{F D M}$, the system sequence knowledge base $J_{\rho}$ is calculated from equation (20). The system sequence constraint matrix $K_{\rho}$ is typically nonzero because it must reflect continuity relations as constraints [65-69]. However, because the transportation structural degrees of freedom have already been eliminated, such constraints no longer apply. Instead, further constraints may arise from logical sequences in the clinical practice of medicine as described by Figure 4. More specifically, a transformation cannot occur immediately after a measurement; a decision must occur in between. This introduces a total of 375 constraints in $K_{\rho}$ which eliminates 15 sequence degrees of freedom. The associated heterofunctional network adjacency matrix $A_{\rho}$ is shown in Figure 7. The associated number of system sequence degrees of freedom is calculated from equation (22). $\mathrm{DOF}_{\rho}=181$.

Returning to the narrative of Case Study 1, it can now be rewritten as a string of healthcare delivery system events $\mathscr{E}_{S}$ as shown in Figure 8. Each event in $\mathscr{E}_{S}$ has a unique index 
TABLE 3: Summary of the analogy between mass-customized productions systems and personalized healthcare delivery.

\begin{tabular}{lcc}
\hline System & Mass-customized production system & Personalized healthcare delivery system \\
\hline (A) System form & & Buffer $\left(R_{B}\right)\left[\right.$ Transformation $\left(R_{F}\right) \cup$ \\
Resources & Buffer $\left(B_{S}\right)$ [Transformation $(M) \cup$ & Decision $\left(R_{D}\right) \cup$ Measurement $\left.\left(R_{M}\right)\right] \cup$ \\
& Independent buffer $(B)] \cup$ & Transportation $\left(R_{N}\right)$ \\
Resource classification & Transportation $(H)$ & Transform $>$ Decide $>$ Measure $>$ \\
& Fixed & Transportation
\end{tabular}

(B) System function

Processes Transformation $\left(P_{\mu}\right) \cup \operatorname{Transportation}\left(P_{\eta}\right)$

Transformation $\left(P_{F}\right) \cup$ Decision $\left(P_{D}\right) \cup$ Measurement $\left(P_{M}\right) \cup$ Transportation $\left(P_{N}\right)$

(C) System concept

System knowledge base

$J_{S}=\left[\begin{array}{ccc}J_{M} & \mid & 0 \\ \longleftarrow & J_{H} & \longrightarrow\end{array}\right]$

$J_{S}=\left[\begin{array}{cccc}J_{F} & 0 & 0 & 0 \\ J_{F D} & J_{D} & 0 & 0 \\ J_{F M} & J_{D M} & J_{M} & 0 \\ J_{F N} & J_{D N} & J_{M N} & J_{N}\end{array}\right]$

System constraint matrix

$K_{S}=\left[\begin{array}{ccc}K_{M} & \mid & 0 \\ \longleftarrow & K_{H} & \longrightarrow\end{array}\right]$

$K_{S}=\left[\begin{array}{cccc}K_{F} & 0 & 0 & 0 \\ K_{F D} & K_{D} & 0 & 0 \\ K_{F M} & K_{D M} & K_{M} & 0 \\ K_{F N} & K_{D N} & K_{M N} & K_{N}\end{array}\right]$

Structural degrees of freedom (nodes)

$\mathrm{DOF}_{S}=\sum_{w}^{\sigma(P)} \sum_{v}^{\sigma(R)}\left[J_{S} \ominus K_{S}\right](w, v)$

$D O F_{S}=\sum_{w}^{\sigma(P)} \sum_{v}^{\sigma(\mathbb{R})}\left[J_{S} \ominus K_{S}\right](w, v)$

System sequence knowledge base

System sequence constraint matrix

System sequence degrees of freedom (edges)

$$
J_{\rho}=\left[J_{S} \cdot \bar{K}_{S}\right]^{V}\left[J_{S} \cdot \bar{K}_{S}\right]^{V T}
$$$$
J_{\rho}=\left[J_{S} \cdot \bar{K}_{S}\right]^{V}\left[J_{S} \cdot \bar{K}_{S}\right]^{V T}
$$

$\operatorname{DOF}_{\rho}=\sum_{\psi_{1}}^{\sigma(P)} \sum_{\psi_{2}}^{\sigma(R)}\left[J_{\rho} \ominus K_{\rho}\right]\left(\psi_{1}, \psi_{2}\right)$

$\mathrm{DOF}_{\rho}=\sum_{\psi_{1}}^{\sigma(P)} \sum_{\psi_{2}}^{\sigma(\mathbb{R})}\left[J_{\rho} \ominus K_{\rho}\right]\left(\psi_{1}, \psi_{2}\right)$

D) Operand Petri-net model

Places

Product net $\left(N_{l_{i}}\right)$

Product places $\left(S_{l_{i}}\right)$

Transitions

Transition duration

Arcs

Arc weight

(E) Coordinating system and operand

Operand Transformation Feasibility Matrix
Product event $\left(\mathscr{E}_{l_{i}}\right)$

Infinitesimal

$$
\mathscr{M}_{l_{i}}
$$

$\{0,1\}$

Production

Product Transformation Feasibility Matrix $\left(\Lambda_{\mu_{i}}\right)$ of size $\sigma\left(\mathscr{E}_{l_{i}}\right) \times \sigma\left(P_{\mu}\right)$
Health net $\left(N_{l_{i}}\right)$

Health state $\left(S_{l_{i}}\right)$

Health event $\left(\mathscr{E}_{l_{i}}\right)$

Fixed duration $\left(D_{l_{i}}\right)$

$$
\mathscr{M}_{l_{i}}
$$

Stochastic ("Fuzzy”) $\left(W_{l_{i}}\right)$

Healthcare

Individual Transformation Feasibility Matrix $\left(\Lambda_{F_{i}}\right)$ of size $\sigma\left(\mathscr{E}_{l_{i}}\right) \times \sigma\left(P_{F}\right)$ and its associated combination of process and resource. The transformational events are highlighted in bold.

3.3. Modeling the Individual Health Net Episode of the Orthopedic Case. From the narrative of Case Study 1 and its associated healthcare delivery system events, the Individual Health Net $N_{l}$ and the Individual Transformation Feasibility Matrix $\Lambda_{F}$ can be constructed as shown in Figures 9 and 10, respectively.

The Health Net shows the individual's health states at the places (circles) and the individual's health state transformations at the transitions (rectangles) which may occur due to the healthcare delivery system events $P_{F}$ or the stochastic human process $P_{\varphi}$.

The Individual Transformation Feasibility Matrix is constructed by linking the Individual Health Net transitions (i.e., health events) to the corresponding Healthcare Delivery System Transformational Events (i.e., transformation process $\left.P_{F}\right)$.

In summary, the Healthcare System Architecture for the acute orthopedic example has been developed and described in terms of the five components in Figure 1. This acute episode case study quantitatively describes the application of this system model for personalized healthcare delivery and managed individual health outcomes. 


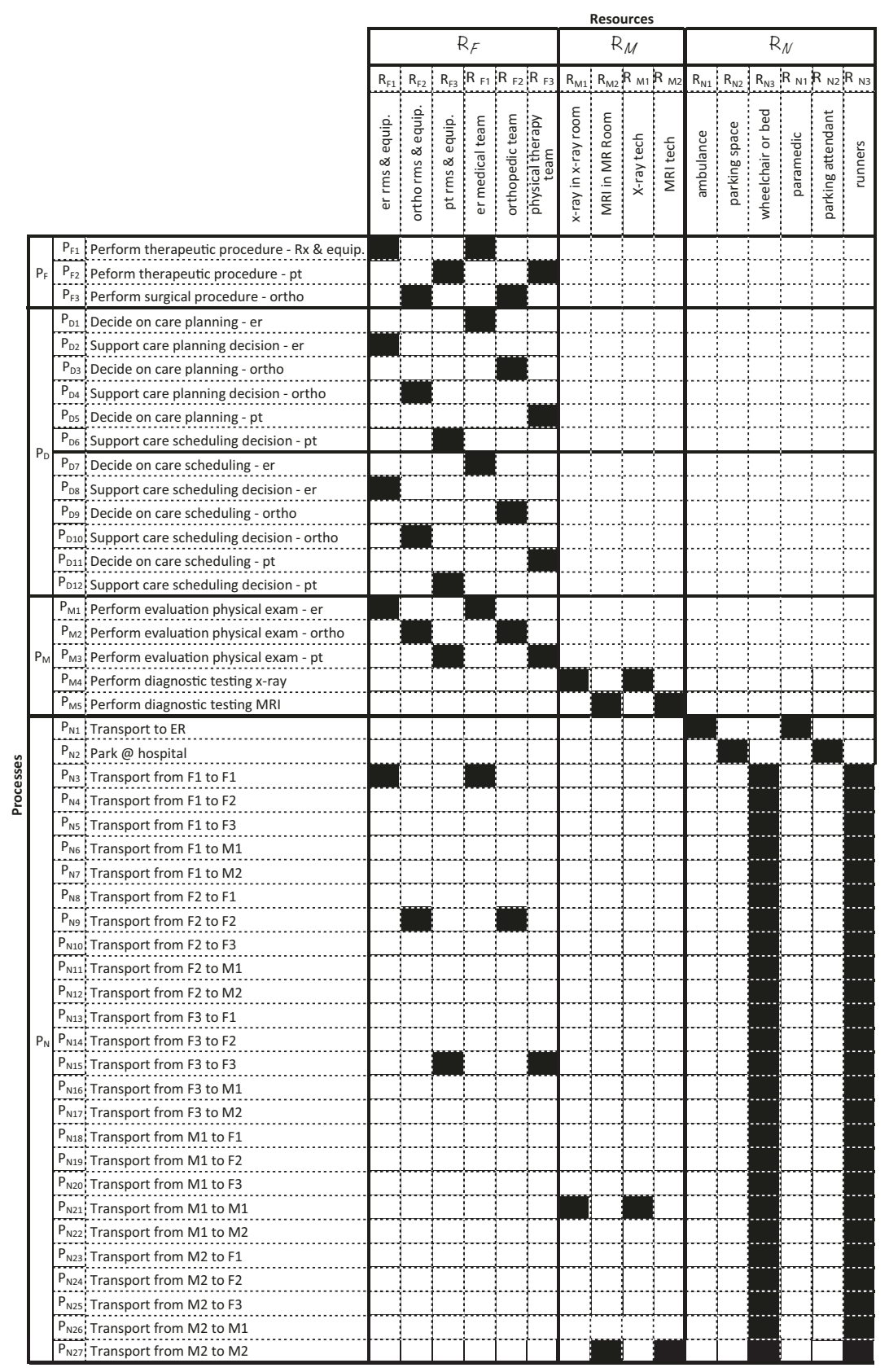

FIGURE 5: Lowest level healthcare delivery system knowledge base $J_{S}$ with allocated processes to resources (dark filled) for the acute orthopedic example.

\section{Chronic Care Illustrative Example}

The system architecture model is used to model a chronic episode of a diabetic case demonstrating the importance of communication between primary and specialized care in the coordinated healthcare of individuals with diabetes. This example specifically illustrates the difference in healthcare transformation processes and individual health state evolution when an episode is misclassified as acute rather than chronic. As before, Sections 4.2 and 4.3 parse this narrative into quantitative models of the Healthcare Delivery System and the Individual's Health State.

4.1. Description of Diabetic Case. An example diabetes case study is described below; it is drawn without modification directly from an example textbook clinical case [116].

Case Study 2. "Juanita is a 66-year old Hispanic individual with a 20 -year history of poorly controlled T1DM, chronic 


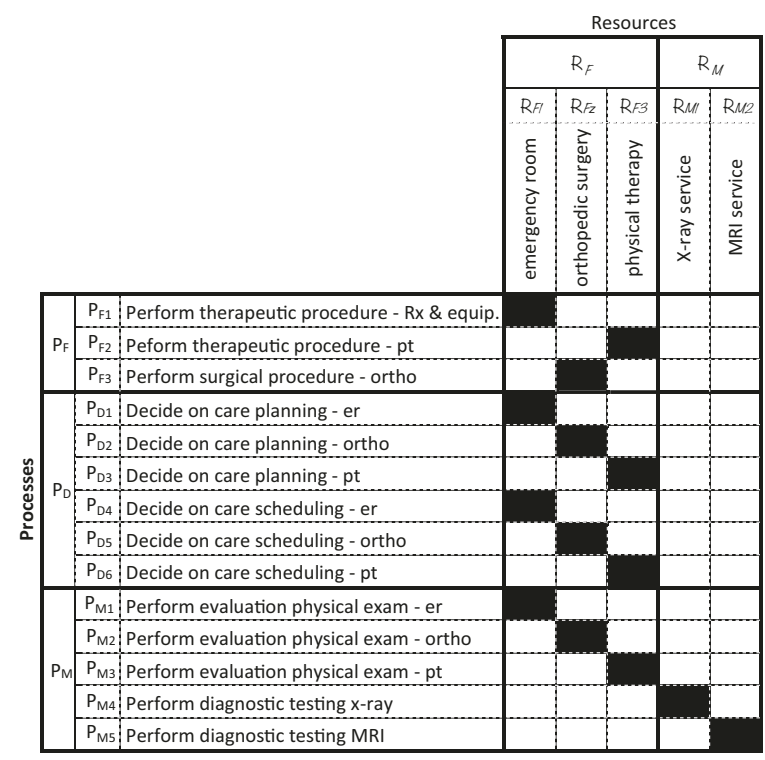

FIGURE 6: Higher level (transformation, decision, and measurement) knowledge base $\bar{J}_{F D M}$ with allocated aggregated processes to aggregated resources (dark filled), for the acute orthopedic example.

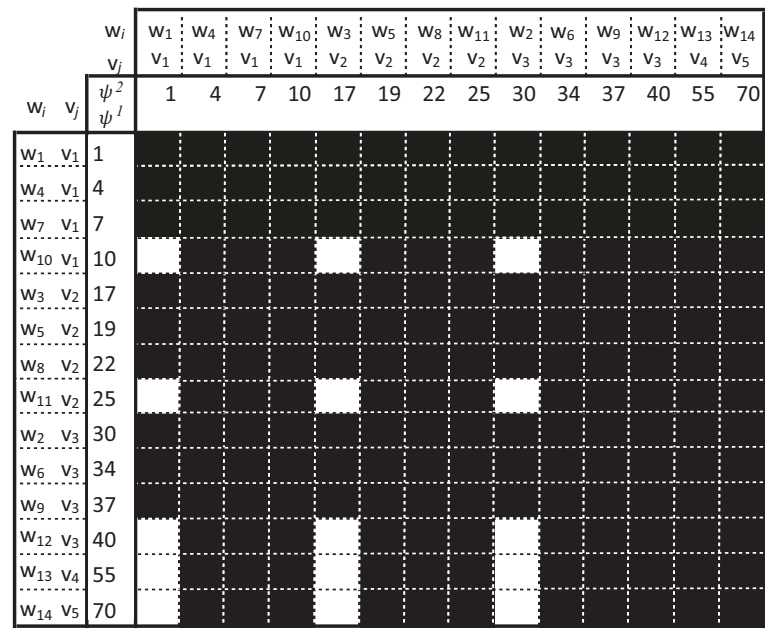

Figure 7: Adjacency matrix $A_{\rho}(\phi, \psi)$ composed of sequence events (dark filled), for the acute orthopedic example.

kidney disease, and diabetic amyotrophy. She decided to consult an orthopedic specialist on her own for "terrible leg pains." After a brief workup, which consisted of a magnetic resonance image (MRI) of the knee, a decision was made to perform a total joint replacement on her arthritic right knee. Although the surgeon considered the procedure a "great success," the individual had persistent pain postoperatively, which actually worsened over a 3-month period. The frustrated surgeon could not understand why the individual was complaining of so much pain "when the bone scan and postoperative x-rays showed no evidence of osteomyelitis." The 12 visits of physical therapy also appeared to worsen her discomfort to the point where she became incapacitated by the pain. She was having increasing difficulty with her balance and could not tolerate having any bed sheets come in contact with her feet. Four months after having her knee replacement surgery, she returned to her PCP.

On examination the individual exhibited hyperalgesia, allodynia, and loss of ankle reflexes bilaterally, which was worse on the right (postoperative) extremity. The individual wore a slipper on the right foot to lessen the effects of her painful peripheral diabetic neuropathic pain. The PCP placed the individual on duloxetine, which resulted in a $50 \%$ improvement in her overall pain intensity within 3 weeks. Communication between the specialist and the PCP is of utmost importance when managing individuals with diabetes. Had the surgeon discussed this case with the PCP prior to operating he would have realized that a more conservative approach was warranted. Not only was this individual's pain the result of diabetic peripheral neuropathy 


\begin{tabular}{|c|c|c|c|c|}
\hline Episode & $\begin{array}{l}\text { DOF } \\
\text { Index }\end{array}$ & $\begin{array}{l}\text { DOF } \\
\text { Event }\end{array}$ & DOF Process & DOF Resource \\
\hline \multirow{16}{*}{$\begin{array}{c}\mathrm{ACL} \\
\text { injury } \\
\& \text { repair }\end{array}$} & 10 & $\mathrm{e}_{\mathrm{M} 1 F 1}$ & Perform evaluation physical exam - er & by emergency room \\
\hline & 13 & $\mathrm{e}_{\mathrm{M} 4 \mathrm{M} 1}$ & 1 Perform diagnostic testing $x$-ray & by X-ray service \\
\hline & 4 & $e_{D 1 F 1}$ & Decide on care planning - er & by emergency room \\
\hline & 7 & $e_{D 4 F 1}$ & Decide on care scheduling - er & by emergency room \\
\hline & 1. & $e_{F 1 F 1}$ & Perform therapeutic procedure - Rx \& equ & by emergency room \\
\hline & 7 & $\mathrm{e}_{\mathrm{D} 4 \mathrm{~F} 1}$ & Decide on care scheduling - er & by emergency room \\
\hline & 11 & $\mathrm{e}_{\mathrm{M} 2 F 2}$ & Perform evaluation physical exam - ortho & by orthopedic surgery \\
\hline & 14 & $\mathrm{e}_{\mathrm{M} 5 \mathrm{M} 2}$ & 2 Perform diagnostic testing MRI & by MRI service \\
\hline & 5 & $\mathrm{e}_{\mathrm{D} 2 \mathrm{~F} 2}$ & Decide on care planning - ortho & by orthopedic surgery \\
\hline & 8 & $e_{D 5 F 2}$ & Decide on care scheduling - ortho & by orthopedic surgery \\
\hline & 3 & $e_{F 3 F 2}$ & Perform surgical procedure - ortho & by orthopedic surgery \\
\hline & 8 & $\mathrm{e}_{\mathrm{DSF2} 2}$ & Decide on care scheduling - ortho & by orthopedic surgery \\
\hline & 12 & $\mathrm{e}_{\mathrm{M} 3 F 3}$ & Perform evaluation physical exam - pt & by physical therapy \\
\hline & 6 & $e_{D 3 F 3}$ & Decide on care planning - pt & by physical therapy \\
\hline & 9 & $\mathrm{e}_{\mathrm{D} 6 \mathrm{~F}}$ & Decide on care scheduling - pt & by physical therapy \\
\hline & 2 & $e_{F 2 F 3}$ & Peform therapeutic procedure - pt & by physical therapy \\
\hline
\end{tabular}

FIGURE 8: Orthopedic acute episode described in terms of the healthcare delivery system events found in $\bar{J}_{F D M}$. The healthcare delivery transformational events are in bold.

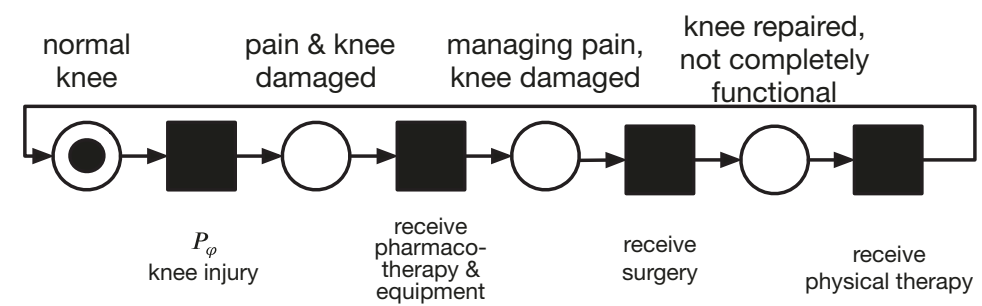

Figure 9: Acute orthopedic Individual Health Net visualizes the health state at the places (circles) and the health events, causing the changes in health state, at the transitions (rectangles).

rather than "arthritis" but her fasting blood glucose level on the day of surgery was $323 \mathrm{mg}$ per dL. The PCP was unaware that the individual was even hospitalized. Had the specialist been concerned about the individual's preoperative laboratory studies (including her A1C of 12.2\%), the surgery would have been canceled until she was medically cleared to undergo the procedure. On the second postoperative day, the individual developed acute renal failure." [116].

This example addresses two possible outcome episodes: (1) Episode A describes the events as they happened (i.e., not taking into account the patient's previous history or that they are currently in a chronic episode) and (2) Episode $B$ describes the hypothetical scenario if appropriate communication between primary and specialty care had been implemented. Such a scenario would take into account the individual's chronic episode and treat it accordingly.

4.2. Modeling the Healthcare Delivery System of the Diabetes Case. Similar to the first example, the modeling of the Healthcare Delivery System Form and Function was determined by identifying the resources and processes in the text of Case Study 2. These were used to construct the system knowledge base $J_{S}$ as shown in Figure 11.
As in the prior case study, the resources and processes at this low-level of abstraction need to be aggregated using equation (4) to a higher level to better reflect the practice of clinical operation. This includes the aggregation of technical and human resources and decision and decision support processes. Transportation capabilities are also assumed to be fully available.

These steps allow the construction of knowledge base $\bar{J}_{F D M}$ at a higher level of aggregation as shown in Figure 12. For simplicity, the system is assumed to not have any event constraints during either episodes. $K_{\mathrm{S}}=0$. The associated number of structural degrees of freedom is calculated from equation (18). $\mathrm{DOF}_{S}=14$.

Continuing with the knowledge base $\bar{J}_{F D M}$, the system sequence knowledge base $J_{\rho}$ is calculated using equation (20). The assumptions made to the system sequence constraint matrix $K_{\rho}$ in the first illustrative example are also applied here. Therefore, continuity relations are discounted and logical clinical practice sequence constraints are applied as described by Figure 4 . This introduces a total of 648 constraints in $K_{\rho}$ which eliminates 18 sequence degrees of freedom. The associated heterofunctional network adjacency matrix $A_{\rho}$ is shown in Figure 13. The associated number of 


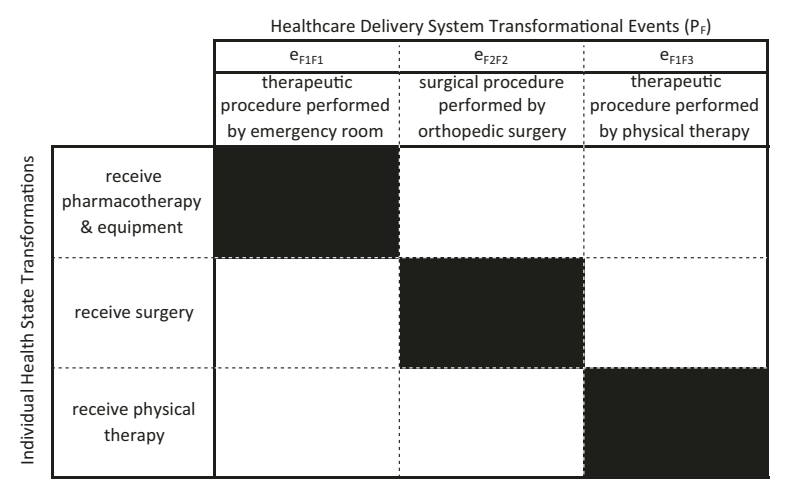

FIgURE 10: Individual Transformation Feasibility Matrix $\Lambda_{F}$ for the acute orthopedic example.

system sequence degrees of freedom is calculated from equation (22). $\mathrm{DOF}_{\rho}=178$.

Returning to the narrative of Case Study 2, it can now be rewritten as a string of healthcare delivery system events $\mathscr{E}_{S}$. Figures 14 and 15 show these strings for Episodes A and B, respectively.

4.3. Modeling the Individual Health Net Episodes of the Diabetic Case. From the diabetes case study narrative and the modeled healthcare delivery system events, the Individual Transformation Feasibility Matrix $\Lambda_{F}$ (see Figure 16) and the Individual Health Net showing both Episodes (see Figure 17) can be constructed. The Individual Health Net Episodes show the individual's health states at the places (circles) and the individual's health state transformations at the transitions (rectangles).

The Individual Transformation Feasibility Matrix is constructed by linking the Individual Health Net transitions (i.e., health events) to the corresponding Healthcare Delivery System Transformational Events (i.e., transformation process $\left.P_{F}\right)$.

In summary, the Healthcare System Architecture for the chronic diabetes example has been developed in terms of the five components in Figure 1. This chronic example case study quantitatively shows the importance of communication in the co-management of chronic diseases. In Episode A, a chronic episode was treated as acute, leading to an adverse effect on the individual's health state evolution. In Episode B, the improved health outcome was achieved through coordinated care.

\section{Discussion}

The acute and chronic care illustrative examples have demonstrated a system model for personalized healthcare delivery and managed individual outcomes. The strengths of the model arise from several network structures that allow for coordinated healthcare while distinguishing between acute and chronic conditions. They are as follows:

(1) The aggregation matrices $A_{R}$ and $A_{P}$.

(2) The system knowledge base $J_{S}$.

(3) The system events constraints matrix $K_{S}$.
(4) The system sequence constraints matrix $K_{\rho}$.

(5) The Health Net $N_{l_{i}}$.

Together, these network structures serve to provide appropriate, coordinated, and personalized healthcare to unique individuals. Furthermore, each of these matrices may be viewed as the outcome of a healthcare delivery system design decision. These decisions are now discussed in the context of the five parts of the healthcare system architecture shown in Figure 1.

The aggregation matrices $A_{R}$ and $A_{P}$ were introduced so as to view the Healthcare Delivery System Form and Function at higher levels of aggregation. The need for physical aggregation reflects how teams of healthcare professionals and groups of technical equipment must often be brought together to form a single operating unit (e.g., surgical team in an operating theatre). Similarly, the need for functional aggregation reflects how many low level system processes are required to perform a single healthcare service (e.g., perform orthopedic surgery). While it is possible to design a healthcare delivery system with constant values of $A_{R}$ and $A_{P}$ such rigidity is prohibitively expensive. Healthcare delivery system administrators must often choose new values of $A_{R}$ so as to form new clinical teams with each shift and assure that the right technical equipment is in the appropriate facilities and rooms. These administrators also choose the values of $A_{P}$ when they formulate hospital procedures in terms of lowlevel system processes.

The system knowledge base $J_{S}$ was introduced so as to view the Healthcare Delivery System Concept in terms of the existence of capabilities that are the feasible combinations of system processes and resources. Fundamentally, it is a succinct description of what the system can do and how. From a design perspective, the value of $J_{S}$ is determined by two types of healthcare delivery system administrators: human resource managers and procurement managers. In hiring new personnel, new columns are added to $J_{S}$. When these new personnel represent new specializations, new rows are added $J_{S}$. Training programs allow each human resource the ability to execute new system processes. Similarly, the procurement of new technical equipment also adds columns to $J_{S}$.

The system events constraints matrix $K_{S}$ was introduced to the Healthcare Delivery System Concept to distinguish 


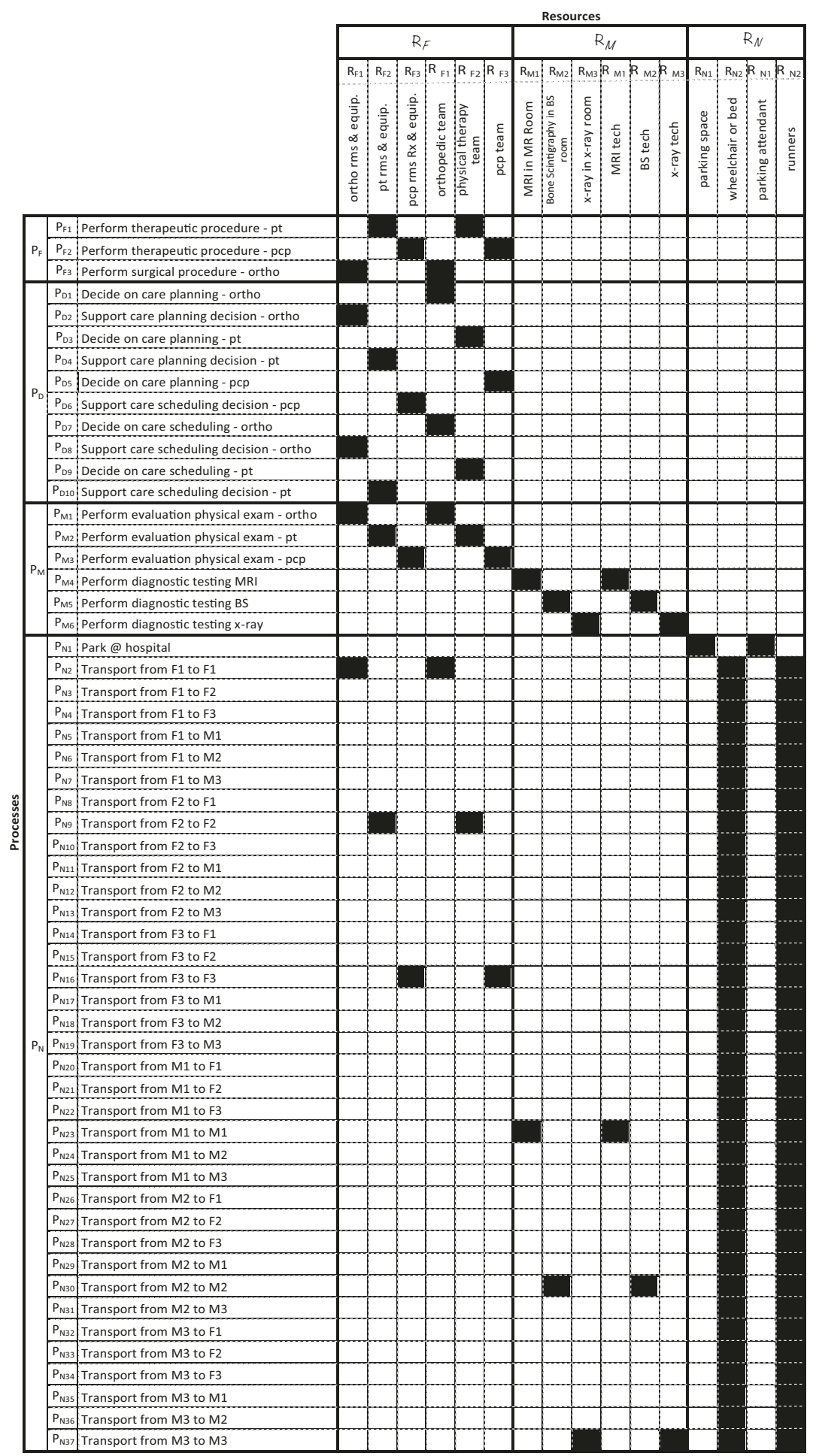

FIGURE 11: Healthcare delivery system knowledge base $J_{S}$ with allocated processes to resources (dark filled) for the chronic diabetes example.

between the existence and the availability of the capabilities. From a design perspective, some availability constraints are planned. These include shift changes for human resources and planned maintenance for technical resources. Other availability constraints may be viewed as unplanned disturbances to the architecture. They include personal and sick leave for human resources and breakdowns for technical resources.

The system sequence constraints matrix $K_{\rho}$ was introduced to the Healthcare Delivery System Concept to distinguish between capabilities as individual elements versus as logical pairs. In the healthcare of acute conditions, where 


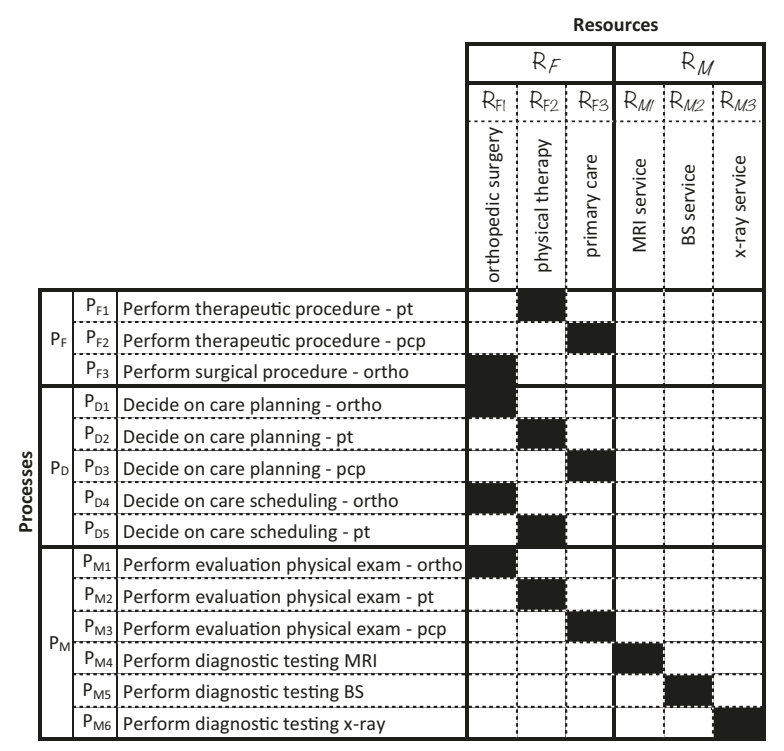

FIGURE 12: Higher level (transformation, decision, and measurement) knowledge base $\bar{J}_{F D M}$ with allocated aggregated processes to aggregated resources (dark filled), for the chronic diabetes example.

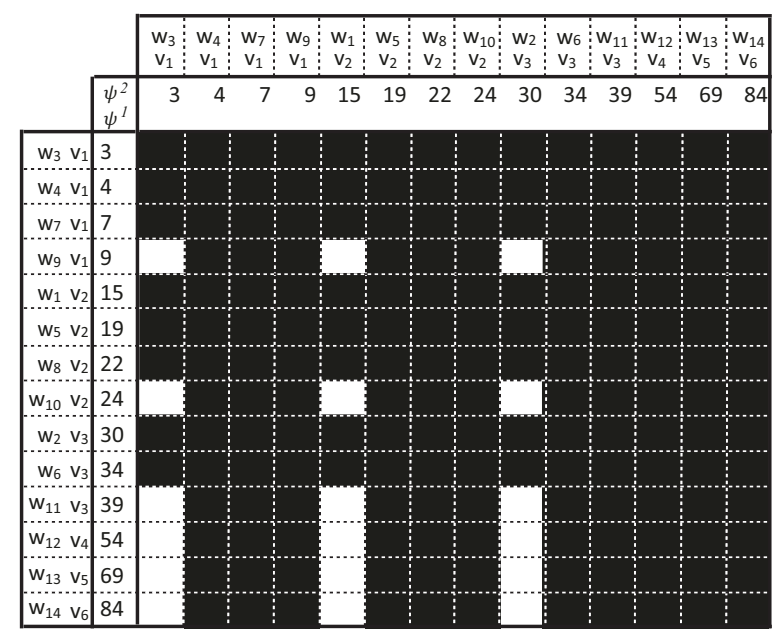

Figure 13: Adjacency matrix $A_{\rho}(\phi, \psi)$ composed of sequence events (dark filled) for the chronic diabetes example.

the timescale is relatively short, continuity relations dominate $K_{\rho}$. Ensuring that patients can move from one "valueadding" healthcare service to another while avoiding lengthy queues is of fundamental importance. Capacity limitations on transportation capabilities are often important. It is for this reason that discrete-event simulation has been featured so prominently in the study of medical emergency healthcare operations [117-120]. In the healthcare of chronic conditions, the timescale is comparatively long. Transportation processes and their associated continuity relations are no longer of prime importance. Instead, sequence-dependent constraints arise from rules of clinical and administrative practice. Clinical practice dictates which types of scans and tests are required for clinical decisions which are required prior to the execution of specific procedures. Such constraints are put in place to assure the quality of medical practice. Further constraints may be placed by healthcare administrators to control costs. These include limitations on the number of scans and clinical consultations.

The Health Net $N_{l_{i}}$ for a given individual $l_{i}$ was introduced as a mathematical description of clinical medical science where the individual's health state requires coordination with the healthcare delivery system in order to achieve the desired health outcomes. In the healthcare of acute conditions, where the timescale is relatively short, health events driven by stochastic human processes $\mathscr{E}_{\varphi l_{i}}$ may not have the chance to occur and so it is reasonable to assume that the health evolution of an individual is purely determined by the health events driven by healthcare transformation processes $\mathscr{E}_{F l_{i}}$. In such a way an individual (patient) becomes a passive entity in 


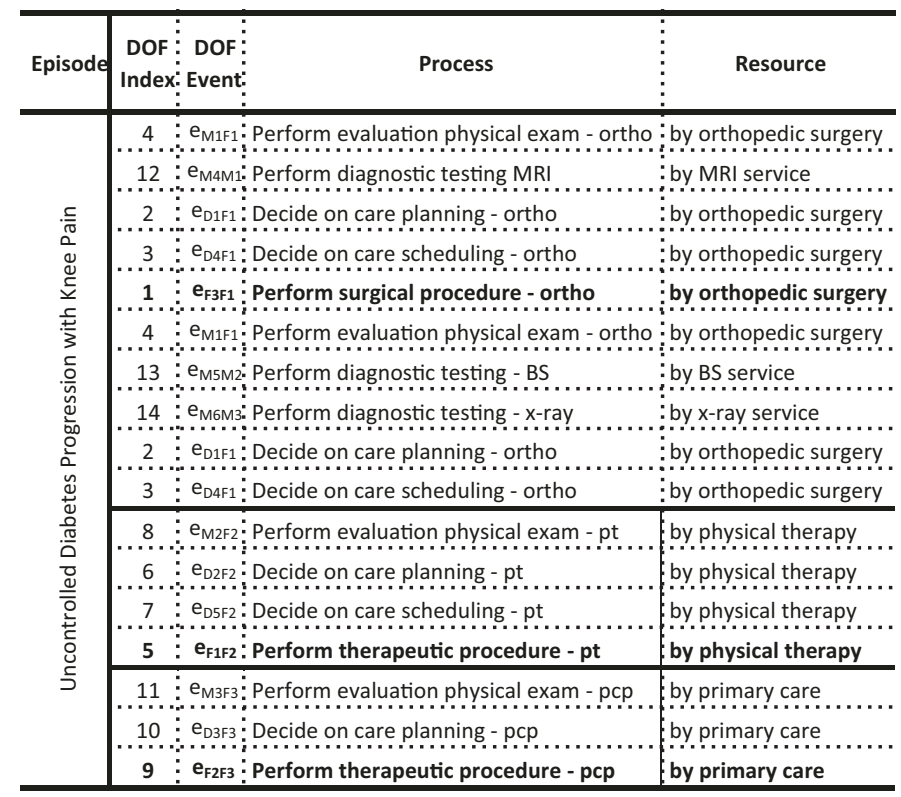

FIGURE 14: Diabetes Chronic Episode A described in terms of the healthcare delivery system events found in $\bar{J}_{F D M}$. The healthcare delivery transformational events are in bold.

\begin{tabular}{|c|c|c|c|c|}
\hline Episode & $\begin{array}{l}\text { DOF } \\
\text { Index }\end{array}$ & $\begin{array}{l}\text { DOF } \\
\text { Event }\end{array}$ & Process & Resource \\
\hline \multirow{3}{*}{ 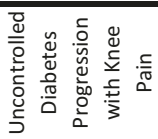 } & 11. & $=3 ;$ & Perform evaluation physical exam - pcp & by primary care \\
\hline & 10 & $\mathrm{e}_{\mathrm{D} 3 F 3}$ & Decide on care planning - $p c p$ & by primary care \\
\hline & 9 & $e_{F 2 F 3}$ & Perform therapeutic procedure - pcp & by primary care \\
\hline
\end{tabular}

Figure 15: Diabetes Chronic Episode B described in terms of the healthcare delivery system events found in $\bar{J}_{F D M}$. The healthcare delivery transformational events are in bold.

the healthcare delivery system. Such is the inherent assumption of many works on discrete-event simulation of medical emergency healthcare operations [117-120]. In contrast, in the healthcare of chronic conditions, the health events driven by stochastic human processes $\mathscr{E}_{\varphi l_{i}}$ play a prominent role and it becomes important to track the evolution of an individual's health state as had been done in mass-customized production systems [65].

That said, an individual's health state has several features that distinguish it from mass-customized products. First, the state of the mass-customized product is typically completely understood and quantifiable, whereas the true state of the human being's health is typically fuzzy. Consequently, the healthcare delivery must heavily utilize measurement processes to ascertain this state, the fundamental reason for the inclusion of measurement processes in the process classification. Second, individuals (or patients) may be viewed as semiautonomous decision-making rather than passive entities [39]. In that regard, the intelligent (mass-customized) product literature [84-86] may prove a relevant extension of the analogy presented in this paper. Recent work has specifically included a product net at the heart of an intelligent product agent's data structure [70] and so one can expect the
Health Net to take a similar role for individuals. As a third distinguishing feature, this model specifically includes decision capabilities because individuals often need to physically meet with clinicians in order for these shared decisions to occur [39].

\section{Conclusion}

In conclusion, this paper architects a system model for personalized healthcare delivery and managed individual health outcomes. This work is built upon recent structural analysis of mass-customized production systems as an analogous system. It highlights the stochastic evolution of an individual's health state as a key distinguishing feature. In doing so, it systematically addressed the new healthcare delivery system requirements described in Table 2 that were derived from healthcare delivery challenges posed by chronic conditions described in Table 1 . The architecture model was then demonstrated for two illustrative examples: one for acute care and another for chronic care. The contrast of the two examples shows inherent complexities of managing personalized healthcare delivery for (potentially multiple) chronic conditions. The development of the architecture model opens 


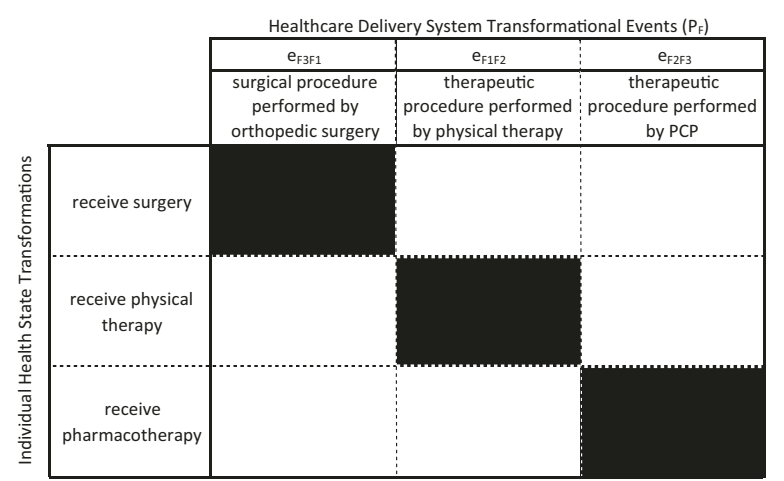

Figure 16: Individual Transformation Feasibility Matrix $\Lambda_{F}$ of the chronic diabetes example.

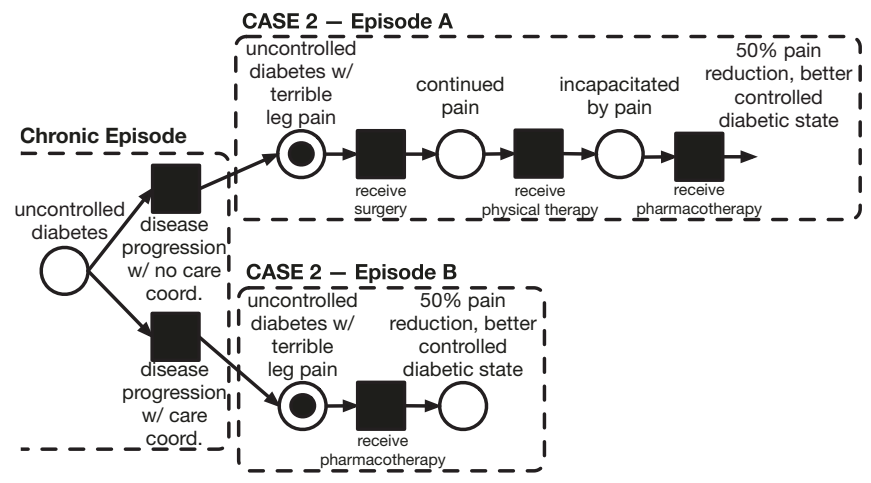

FIGURE 17: Chronic diabetes Individual Health Net illustrating two possible episodes (A or B) based on level of care coordination when disease progresses for an individual in a chronic episode. Health Net visualizes health states at the places (circles) and health state transformations, causing the changes in health state, at the transitions (rectangles).

several avenues for future work including discrete-event simulation, resilience analysis, and optimization methods.

The developed model directly addresses the complexity arising from treating chronic diseases and in doing so incorporates the stochastic evolution of an individual's health in relation to the healthcare delivery system. This is in contrast to classic healthcare simulation of an individual as a stateless passive entity. From a healthcare management perspective, such a model architecture captures two key shifts in healthcare: (1) the shift towards allowing human resources to "practice at the top of their license" and (2) taking into consideration patient preferences in the quickly rising literature of shared decision-making.

\section{Conflicts of Interest}

The authors declare that they have no conflicts of interest.

\section{References}

[1] R. Uzsoy, "Supply-chain management and health care delivery: Pursuing a system-level understanding," Building a Better Delivery System: A New Engineering/Health Care Partnership, pp. 143-146, 2005.
[2] R. R. McDaniel Jr. and D. J. Driebe, "Complexity science and health care management," Advances in Health Care Management, vol. 2, pp. 11-36, 2001.

[3] P. E. Plsek and T. Greenhalgh, "Complexity science: the challenge of complexity in health care," BMJ: British Medical Journal, vol. 323, no. 7313, pp. 625-628, 2001.

[4] P. E. Plsek and T. Greenhalgh, "The challenge of complexity in health care," BMJ: British Medical Journal, vol. 323, no. 7313, pp. 625-628, 2001, http://www.ncbi.nlm.nih.gov/pmc/articles/ PMC1121189/.

[5] C. Gershenson, "Harnessing the complexity of education with information technology," Complexity, vol. 20, no. 5, pp. 13-16, 2015.

[6] E. H. Wagner, B. T. Austin, C. Davis, M. Hindmarsh, J. Schaefer, and A. Bonomi, "Improving chronic illness care: translating evidence into action," Health Affairs, vol. 20, no. 6, pp. 64-78, 2001.

[7] R. D. C. Hoffman, Chronic Care in America: A 21st Century Challenge, Robert Wood Johnson Foundation, 1996, https:// books.google.com/books?id=EklrAAAAMAAJ.

[8] D. T. Wade and P. W. Halligan, "Do biomedical models of illness make for good healthcare systems?" British Medical Journal, vol. 329, no. 7479, pp. 1398-1401, 2004.

[9] R. Porter, "The Greatest benefit to mankind: a medical history of humanity," in The Norton History of Science, W. W. Norton \& Company, 1999. 
[10] G. L. Engel, “The need for a new medical model: a challenge for biomedicine," Science, vol. 196, no. 4286, pp. 129-136, 1977.

[11] G. L. Engel, "The biopsychosocial model and the education of health professionals," General Hospital Psychiatry, vol. 1, no. 2, pp. 156-165, 1979.

[12] R. Feo and A. Kitson, "Promoting patient-centred fundamental care in acute healthcare systems," International Journal of Nursing Studies, vol. 57, pp. 1-11, 2016, http://www..sciencedirect.com/science/article/pii/S0020748916000079.

[13] A. C. Ahn, M. Tewari, C.-S. Poon, and R. S. Phillips, "The limits of reductionism in medicine: Could systems biology offer an alternative?" PLoS Medicine, vol. 3, no. 6, pp. 0709-0713, 2006.

[14] A. Alwan, Global Status Report on Noncommunicable Diseases, World Health Organization, Geneva, Switzerland, 2010, http:// whqlibdoc.who.int/publications/2011/9789240686458_eng.pdf.

[15] C. J. L. Murray, T. Vos, and R. Lozano, "Disability-adjusted life years (DALYs) for 291 diseases and injuries in 21 regions, 19902010: A systematic analysis for the Global Burden of Disease Study," The Lancet, vol. 380, no. 9859, pp. 2197-2223, 2012.

[16] B. W. Ward, J. S. Schiller, and R. A. Goodman, "Multiple chronic conditions among us adults: a 2012 update," Preventing Chronic Disease, vol. 11, no. 4, Article ID 130389, p. E62, 2014, http://dx.doi.org/10.5888/pcd11.130389.

[17] S. J. Olshansky, D. J. Passaro, R. C. Hershow et al., "A potential decline in life expectancy in the United States in the 21st century," New England Journal of Medicine, vol. 352, no. 11, pp. 1138-1145, 2005.

[18] J. Gerteis, D. Izrael, D. Deitz et al., Multiple chronic conditions chartbook, 2014.

[19] G. Anderson and J. Horvath, "The growing burden of chronic disease in America," Public Health Reports, vol. 119, no. 3, pp. 263-270, 2004.

[20] R. A. Goodman, S. F. Posner, E. S. Huang, A. K. Parekh, and H. K. Koh, "Defining and measuring chronic conditions: imperatives for research, policy, program, and practice," Preventing Chronic Disease, vol. 10, Article ID 120239, 2013.

[21] M. T. McKenna, W. R. Taylor, J. S. Marks, and J. P. Koplan, "Current issues and challenges in chronic disease control," in Chronic Disease Epidemiology and Control, vol. 2, pp. 1-26, 1998.

[22] A. B. Bernstein, "Health care in America: Trends in utilization," in US Department of Health and Human Services, Centers for Disease Control and Prevention, National Center for Health Statistics, 2004.

[23] W. Hwang, W. Weller, H. Ireys, and G. Anderson, "Out-ofpocket medical spending for care of chronic conditions," Health Affairs, vol. 20, no. 6, pp. 267-278, 2001.

[24] G. Warshaw, "Introduction: Advances and challenges in care of older people with chronic illness," Generations, vol. 30, no. 3, pp. 5-10, 2006.

[25] B. Friedman, H. J. Jiang, and A. Elixhauser, "Costly hospital readmissions and complex chronic illness," Inquiry: The Journal of Health Care Organization, Provision, and Financing, vol. 45, no. 4, pp. 408-421, 2008.

[26] US Department of Health and Human Services, "Multiple chronic conditions-a strategic framework: optimum health and quality of life for individuals with multiple chronic conditions," Tech. Rep., US Department of Health and Human Services, 2010.

[27] G. F. Anderson, Chronic care: making the case for ongoing care, Robert Wood Johnson Foundation, 2010.
[28] US Department of Health and Human Services, "Multiple chronic conditions-a strategic framework: optimum health and quality of life for individuals with multiple chronic conditions, US Department of Health and Human Services, Washington, DC, USA, 2010.

[29] J. L. Haggerty, R. J. Reid, G. K. Freeman, B. H. Starfield, C. E. Adair, and R. McKendry, "Continuity of care: a multidisciplinary review," British Medical Journal, vol. 327, no. 7425, pp. 1219-1221, 2003.

[30] M. D. Cabana and S. H. Jee, "Does continuity of care improve patient outcomes," J Fam Pract, vol. 53, no. 12, pp. 974-980, 2004.

[31] G. Van Servellen, M. Fongwa, and E. Mockus D’Errico, “Continuity of care and quality care outcomes for people experiencing chronic conditions: A literature review," Nursing \& Health Sciences, vol. 8, no. 3, pp. 185-195, 2006.

[32] D. Falvo, "Medical and psychosocial aspects of chronic illness and disability," Jones \& Bartlett Publishers, 2013.

[33] J. S. House, "Understanding social factors and inequalities in health: 20th century progress and 21st century prospects," Journal of Health and Social Behavior, vol. 43, no. 2, pp. 125-142, 2002.

[34] R. A. Aronowitz, Making sense of illness: Science, society and disease, Cambridge University Press, 1998.

[35] Centers for Disease Control and Prevention, "Chronic Disease Overview FactSheet," http://www.cdc.gov/chronicdisease/ overview/index.htm.

[36] D. Jones and S. Quinn, Textbook for Functional Medicine, The Institute for Functional Medicine, Gig Harbor, WA, USA, 2010.

[37] F. Borell-Carrió, A. L. Suchman, and R. M. Epstein, “The biopsychosocial model 25 years later: principles, practice, and scientific inquiry," Annals of Family Medicine, vol. 2, no. 6, pp. 576-582, 2004.

[38] Institute of Medicine, Crossing the Quality Chasm, National Academies Press, Washington, DC, USA, 2001.

[39] A. Edwards and G. Elwyn, Shared Decision-Making in Health Care: Achieving Evidence-Based Patient Choice, Oxford University Press, 2009.

[40] Z. Laron, A. Galatzer, S. Amir, R. Gil, M. Karp, and M. Mimouni, "A multidisciplinary, comprehensive, ambulatory treatment scheme for diabetes mellitus in children," Diabetes Care, vol. 2, no. 4, pp. 342-347, 1979.

[41] V. Dargis, O. Pantelejeva, A. Jonushaite, L. Vileikyte, and A. J. M. Boulton, "Benefits of a multidisciplinary approach in the management of recurrent diabetic foot ulceration in Lithuania: A prospective study," Diabetes Care, vol. 22, no. 9, pp. 1428-1431, 1999.

[42] D. Tripathy, "Multidisciplinary care for breast cancer: Barriers and solutions," Breast Journal, vol. 9, no. 1, pp. 60-63, 2003.

[43] M. L. Fennell, I. P. Das, S. Clauser, N. Petrelli, and A. Salner, “The organization of multidisciplinary care teams: Modeling internal and external influences on cancer care quality," Journal of the National Cancer Institute - Monographs, no. 40, pp. 72-80, 2010.

[44] E. H. Wagner, B. T. Austin, and M. von Korff, "Organizing care for patients with chronic illness," The Milbank Quarterly, vol. 74, no. 4, pp. 511-544, 1996, http://www.jstor.org/stable/3350391.

[45] W. Katon, M. Von Korff, E. Lin et al., "Collaborative Management to Achieve Treatment Guidelines: Impact on Depression in Primary Care," JAMA: The Journal of the American Medical Association, vol. 273, no. 13, pp. 1026-1031, 1995. 
[46] K. Strosahl, "Integrating behavioral health and primary care services: The primary mental health care model," in APA PsycNet, W. W. Norton \& Company, 1998.

[47] S. M. Shortell, F. M. Wu, V. A. Lewis, C. H. Colla, and E. S. Fisher, "A taxonomy of accountable care organizations for policy and practice," Health Services Research, vol. 49, no. 6, pp. 1883-1899, 2014.

[48] P. E. Plsek and T. Wilson, "Complexity science: Complexity, leadership, and management in healthcare organisations," $B M J$, vol. 323, no. 7315, pp. 746-749, 2001, http://www.ncbi.nlm.nih .gov/pmc/articles/PMC1121291/.

[49] B. J. Pine, Mass Customization: The New Frontier in Business Competition, Harvard Business Press, 1993.

[50] M. G. Mehrabi, A. G. Ulsoy, Y. Koren, and P. Heytler, “Trends and perspectives in flexible and reconfigurable manufacturing systems," Journal of Intelligent Manufacturing, vol. 13, no. 2, pp. 135-146, 2002.

[51] S. L. Goldman, "Agile Competitors and Virtual Organizations: Strategies for Enriching the Customer," Van Nostrand Reinhold Company, 1995.

[52] D. C. McFarlane and S. Bussmann, "Holonic manufacturing control: rationales, developments and open issues," in Agentbased manufacturing, pp. 303-326, Springer, 2003.

[53] R. F. Babiceanu and F. F. Chen, "Development and applications of holonic manufacturing systems: A survey," Journal of Intelligent Manufacturing, vol. 17, no. 1, pp. 111-131, 2006.

[54] R. Brennan and D. Norrie, "Agents, holons and function blocks: Distributed intelligent control in manufacturing," Journal of Applied Systems Science: Special Issue, vol. 2, no. 1, p. 19, 2001.

[55] A. I. Dashchenko, Reconfigurable Manufacturing Systems and Transformable Factories, Springer, Berlin, Germany, 2006.

[56] A. M. Farid, A Review of Holonic Manufacturing Systems Literature, University of Cambridge Institute for Manufacturing, Cambridge, UK, 2004.

[57] P. Leitao, "Agent-based distributed manufacturing control: a state-of-the-art survey," Engineering Applications of Artificial Intelligence, vol. 22, no. 7, pp. 979-991, 2009.

[58] V. Marik, M. Fletcher, M. Pechoucek et al., "Holons \& Agents: Recent Developments and Mutual Impacts," in Multi-Agent Systems and Applications II, vol. 2322 of Lecture Notes in Computer Science, pp. 233-267, Springer, Berlin, Germany, 2002.

[59] D. C. McFarlane and S. Bussmann, "Developments in holonic production planning and control," Production Planning and Control, vol. 11, no. 6, pp. 522-536, 2000.

[60] "Holonic manufacturing control: rationales, developments and open issues," in Agent-Based Manufacturing, vol. ch. 13, pp. 303326, Springer-Verlag, Berlin, Germany, 2003.

[61] R. Setchi and N. Lagos, "Reconfigurability and reconfigurable manufacturing systems state-of-the-art review," in Innovative Production Machines and Systems: Production Automation and Control State of the Art Review, vol. ch. 5 of Innovative Production Machines and Systems: Network of Excellence, pp. 131-143, Cardiff, UK.

[62] W. Shen and D. H. Norrie, "Agent-Based Systems for Intelligent Manufacturing: A State-of-the-Art Survey," Knowledge and Information Systems: An International Journal, vol. 1, no. 2, pp. 129-156, 1999.

[63] W. Shen, D. Norrie, and J. Barthès, MultiAgent Systems for Concurrent Intelligent Design and Manufacturing, Taylor \& Francis, Abingdon, UK, 2000.
[64] W. Shen, Q. Hao, H. J. Yoon, and D. H. Norrie, "Applications of agent-based systems in intelligent manufacturing: an updated review," Advanced Engineering Informatics, vol. 20, no. 4, pp. 415-431, 2006, http://www.sciencedirect.com/science/article/ pii/S1474034606000292.

[65] A. M. Farid, Reconfigurability measurement in automated manufacturing systems [Ph.D. thesis], University of Cambridge, 2007.

[66] A. M. Farid and D. C. Mcfarlane, "Production degrees of freedom as manufacturing system reconfiguration potential measures," Proceedings of the Institution of Mechanical Engineers, Part B: Journal of Engineering Manufacture, vol. 222, no. 10, pp. 1301-1314, 2008.

[67] A. M. Farid, "Product Degrees of Freedom as Manufacturing System Reconfiguration Potential Measures," in International Transactions on Systems Science and Applications - invited paper, vol. 4, pp. 227-242, 3rd edition, 2008.

[68] A. M. Farid, "Static Resilience of Large Flexible Engineering Systems: Axiomatic Design Model and Measures," IEEE Systems Journal, pp. 1-12, 2015, http://amfarid.scripts.mit.edu/resources/Journals/IES-J19.pdf.

[69] A. M. Farid, "An axiomatic design approach to production path enumeration in reconfigurable manufacturing systems," in Proceedings of the 2013 IEEE International Conference on Systems, Man, and Cybernetics, SMC 2013, pp. 3862-3869, gbr, October 2013.

[70] A. M. Farid and L. Ribeiro, "An Axiomatic Design of a Multiagent Reconfigurable Mechatronic System Architecture," IEEE Transactions on Industrial Informatics, vol. 11, no. 5, pp. 11421155, 2015, http://dx.doi.org/10.1109/TII.2015.2470528.

[71] W. C. Schoonenberg and A. M. Farid, "A dynamic model for the energy management of microgrid-enabled production systems," Journal of Cleaner Production, vol. 164, pp. 816-830, 2017.

[72] I. S. Khayal and A. M. Farid, "The Need for Systems Tools in the Practice of Clinical Medicine," Systems Engineering, vol. 20, no. 1, pp. 3-20, 2017, http://dx.doi.org/10.1002/sys.21374.

[73] I. S. Khayal and A. M. Farid, "The application of model-based systems engineering to the practice of clinical medicine," in Proceedings of the 2017 Annual IEEE International Systems Conference (SysCon), pp. 1-6, Montreal, Canada, April 2017.

[74] M. M. Günal and M. Pidd, "Discrete event simulation for performance modelling in health care: A review of the literature," Journal of Simulation, vol. 4, no. 1, pp. 42-51, 2010.

[75] S. H. Jacobson, S. N. Hall, and J. R. Swisher, "Discrete-event simulation of health care systems," in Patient Flow: Reducing Delay in Healthcare Delivery, vol. 91 of International Series in Operations Research \& Management Science, pp. 211-252, Springer, New York, NY, USA, 2006.

[76] D. M. Buede, The Engineering Design of Systems: Models and Methods, vol. 55, John Wiley \& Sons, 2011.

[77] E. Crawley, B. Cameron, and D. Selva, System Architecture: Strategy and Product Development for Complex Systems, Prentice Hall Press, 2015.

[78] T. Weilkiens, "Systems engineering with SysML/UML: modeling, analysis, design," Morgan Kaufmann, 2011.

[79] S. Friedenthal, A. Moore, and R. Steiner, "A practical guide to SysML: the systems modeling language," Morgan Kaufmann, 2014.

[80] M. Newman, Networks: An Introduction, Oxford University Press, Oxford, UK, 2010.

[81] M. Van Steen, "Graph theory and complex networks: an introduction," vol. 144, Maareen van Steen, 2010. 
[82] C. G. Cassandras, "Introduction to discrete event systems," Springer Science \& Business Media, 2008.

[83] I. S. Khayal and A. M. Farid, "Axiomatic Design Based Human Resources Management for the Enterprise Transformation of the Abu Dhabi Healthcare Labor Pool," Journal of Enterprise Transformation, vol. 5, no. 3, pp. 162-191, 2015, http://dx.doi.org/ 10.1080/19488289.2015.1056449.

[84] D. Mcfarlane, V. Agarwal, A. A. Zaharudin, C. Y. Wong, R. Koh, and Y. Kang, "The Intelligent Product Driven Supply Chain," in Proceedings of the Systems, Man and Cybernetics, 2002 IEEE International Conference, vol. 4, pp. 393-398, 2002.

[85] G. G. Meyer, K. Främling, and J. Holmström, "Intelligent Products: A survey," Computers in Industry, vol. 60, no. 3, pp. 137$148,2009$.

[86] D. McFarlane, V. Giannikas, A. C. Y. Wong, and M. Harrison, "Product intelligence in industrial control: Theory and practice," Annual Reviews in Control, vol. 37, no. 1, pp. 69-88, 2013.

[87] O. de Weck, D. Roos, and C. Magee, Engineering Systems: Meeting Human Needs in A Complex Technological World, MIT Press, Cambridge, Mass, USA, 2011, http://www.knovel.com/ knovel2/Toc.jsp?BookID=4611, http://mitpress-ebooks.mit.edu/ product/engineering-systems.

[88] J. L. Buckingham, E. P. Donatelle, A. Thomas Jr., and J. E. Scherger, Family Medicine: Principles and Practice, Springer Science \& Business Media, 2013.

[89] G. Pahl, W. Beitz, and K. Wallace, Engineering Design: A Systematic Approach, Springer-Verlag, 1996.

[90] N. P. Suh, Axiomatic Design: Advances and Applications, Oxford University Press, 2001.

[91] A. M. Farid and N. P. Suh, Axiomatic Design in Large Systems: Complex Products, Buildings and Manufacturing Systems, Springer, Berlin, Germany, 2016, http://www.springer.com/us/ book/9783319323879.

[92] A. M. Farid, "An engineering systems introduction to axiomatic design," in Axiomatic Design in Large Systems: Complex Products, Buildings \&amp; Manufacturing Systems, A. M. Farid and and N. P. Suh, Eds., pp. 1-47, Springer, Berlin, Germany, 2016, http://dx.doi.org/10.1007/978-3-319-32388-6.

[93] A. Viswanath, E. E. S. Baca, and A. M. Farid, "An axiomatic design approach to passenger itinerary enumeration in reconfigurable transportation systems," IEEE Transactions on Intelligent Transportation Systems, vol. 15, no. 3, pp. 915-924, 2014.

[94] A. Viswanath and A. M. Farid, "A hybrid dynamic system model for the assessment of transportation electrification," in Proceedings of the American Control Conference (ACC '14), pp. 1-7, IEEE, Portland, OR, USA, June 2014, http://dx.doi.org/ 10.1109/ACC.2014.6858810.

[95] "A Hybrid Dynamic System Assessment Methodology for Multi-Modal Transportation-Electrification," IEEE Transactions on Control System Technology, vol. 1, no. 1, pp. 1-12, 2016, http://dx.doi.org/10.1109/TCST.2016.2579602.

[96] W. N. Lubega and A. M. Farid, "A Reference System Architecture for the Energy-Water Nexus," IEEE Systems Journal, vol. 10, no. 1, pp. 1-11, 2014, http://dx.doi.org/10.1109/JSYST.2014 .2302031 .

[97] W. N. Lubega and A. M. Farid, "Quantitative Engineering Systems Model \&amp; Analysis of the Energy-Water Nexus," Applied Energy, vol. 135, no. 1, pp. 142-157, 2014, http:// dx.doi.org/10.1016/j.apenergy.2014.07.101.

[98] A. M. Farid, "Multi-agent system design principles for resilient coordination control of future power systems," Intelligent Industrial Systems, vol. 1, no. 3, pp. 255-269, 2015.
[99] F. K. Pil and M. Holweg, "Linking product variety to orderfulfillment strategies," Interfaces, vol. 34, no. 5, pp. 394-403, 2004.

[100] World Health Organization, International statistical classification of diseases and health related problems (The) ICD-10, 2004.

[101] H. Kitano, Foundations of Systems Biology, MIT press, Cambridge, UK, 2001.

[102] H. Kitano, "Systems biology: a brief overview," Science, vol. 295, no. 5560, pp. 1662-1664, 2002.

[103] C. Sailer and S. Wasner, Differential Diagnosis Pocket, Pocket (Borm Bruckmeier Publishing) Series, Bruckmeier Publishing, LLC, 2003, https://books.google.com/books?id=BFk8AgAACAAJ.

[104] A. T. Raftery, E. K. S. Lim, and A. J. K. Ostor, Churchill's Pocketbook of Differential Diagnosis, Churchill Pocketbooks, Elsevier Health Sciences, UK, 2014, https://books.google.com/books?id= W_1sAwAAQBAJ.

[105] American Diabetes Association, "Classification and Diagnosis of Diabetes," Diabetes Care, vol. 39, pp. S13-S22, 2015, http:// care.diabetesjournals.org/content/39/Supplement_1/S13.abstract.

[106] E. Senkus, S. Kyriakides, S. Ohno et al., "Primary breast cancer: ESMO Clinical Practice Guidelines for diagnosis, treatment and follow-up," Annals of Oncology, vol. 26, Article ID mdv298, pp. v8-v30, 2015.

[107] D. N. Louis, H. Ohgaki, O. D. Wiestler et al., "The 2007 WHO classification of tumours of the central nervous system," Acta Neuropathologica, vol. 114, no. 2, pp. 97-109, 2007.

[108] C. W. Yancy, M. Jessup, B. Bozkurt et al., "2013 ACCF/AHA Guideline for the Management of Heart FailureA Report of the American College of Cardiology Foundation/American Heart Association Task Force on Practice Guidelines," Journal of the American College of Cardiology, vol. 62, no. 16, pp. e147-e239, 2013, http://dx.doi.org/10.1016/j.jacc.2013.05.019.

[109] W. Pedrycz and H. Camargo, "Fuzzy timed Petri nets," Fuzzy Sets and Systems. An International Journal in Information Science and Engineering, vol. 140, no. 2, pp. 301-330, 2003.

[110] Z. Ding, H. Bunke, M. Schneider, and A. Kandel, "Fuzzy timed petri net definitions, properties, and applications," Mathematical and Computer Modelling, vol. 41, no. 2-3, pp. 345360, 2005, http://www.sciencedirect.com/science/article/pii/ S0895717705000853.

[111] Z. Ding, H. Bunke, O. Kipersztok, M. Schneider, and A. Kandel, "Fuzzy timed Petri nets-analysis and implementation," Mathematical and Computer Modelling, vol. 43, no. 3-4, pp. 385-400, 2006.

[112] L. Popova-Zeugmann, Time and Petri Nets, Springer, Heidelberg, Germany, 2013.

[113] McGraw-Hill., "Concise Dictionary of Modern Medicine," 2002.

[114] A. M. Farid, "Measures of reconfigurability and its key characteristics in intelligent manufacturing systems," Journal of Intelligent Manufacturing, vol. 28, no. 2, pp. 353-369, 2017, http:// dx.doi.org/10.1007/s10845-014-0983-7.

[115] L. J. Guthrie, Clinical Case Studies in Physiotherapy: A Guide for Students and Graduates, Elsevier Health Sciences, UK, 2008.

[116] J. Unger, Diabetes Management in Primary Care, LWW Doody's all reviewed collection, Wolters Kluwer Health/Lippincott Williams \& Wilkins, 2007, https://books.google.com/books?id= 7SVFDF0_vE0C.

[117] N. R. Hoot, L. J. LeBlanc, I. Jones et al., "Forecasting Emergency Department Crowding: A Discrete Event Simulation," Annals of Emergency Medicine, vol. 52, no. 2, pp. 116-125, 2008. 
[118] G. R. Hung, S. R. Whitehouse, C. O’Neill, A. P. Gray, and N. Kissoon, "Computer modeling of patient flow in a pediatric emergency department using discrete event simulation," Pediatric Emergency Care, vol. 23, no. 1, pp. 5-10, 2007.

[119] A. Komashie and A. Mousavi, "Modeling emergency departments using discrete event simulation techniques," in Proceedings of the 2005 Winter Simulation Conference, pp. 2681-2685, December 2005.

[120] L. G. Connelly and A. E. Bair, "Discrete event simulation of emergency department activity: a platform for system-level operations research," Academic Emergency Medicine, vol. 11, no. 11, pp. 1177-1185, 2004. 


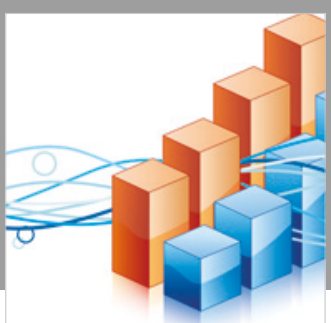

Advances in

Operations Research

\section{-n-m}
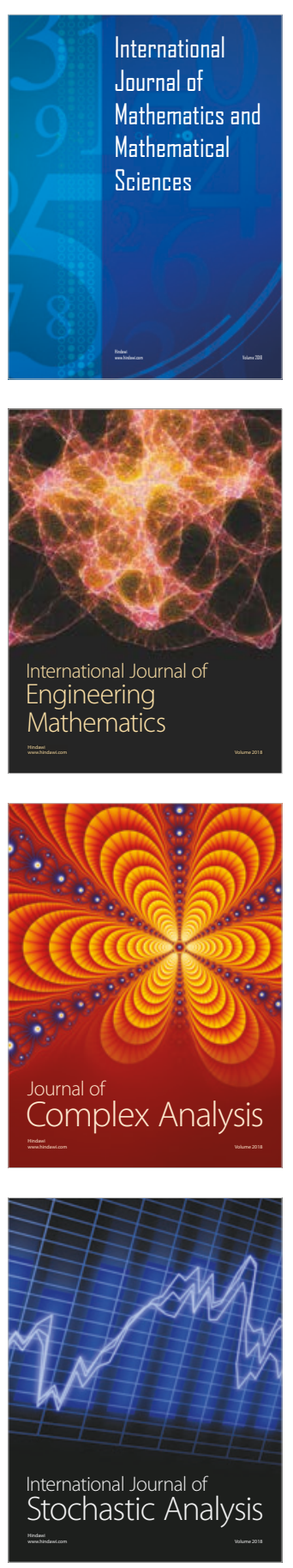
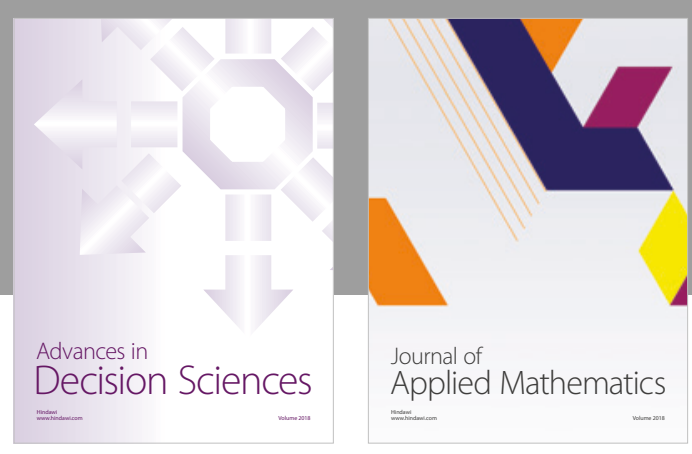

Journal of

Applied Mathematics
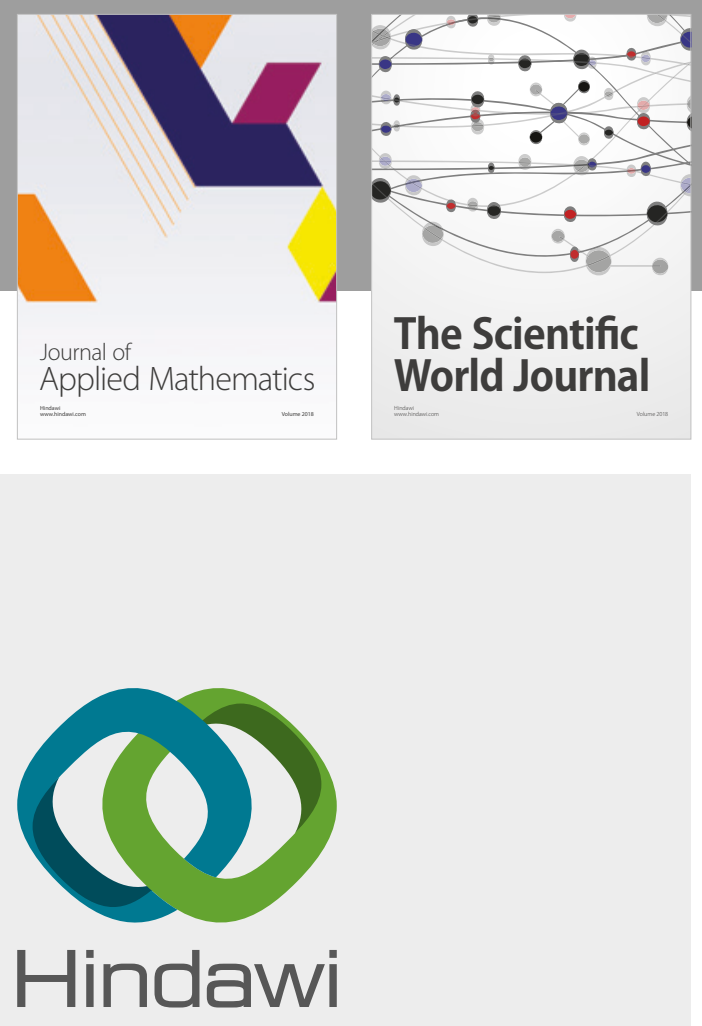

Submit your manuscripts at

www.hindawi.com

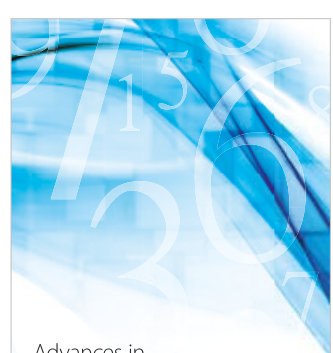

Advances in
Numerical Analysis
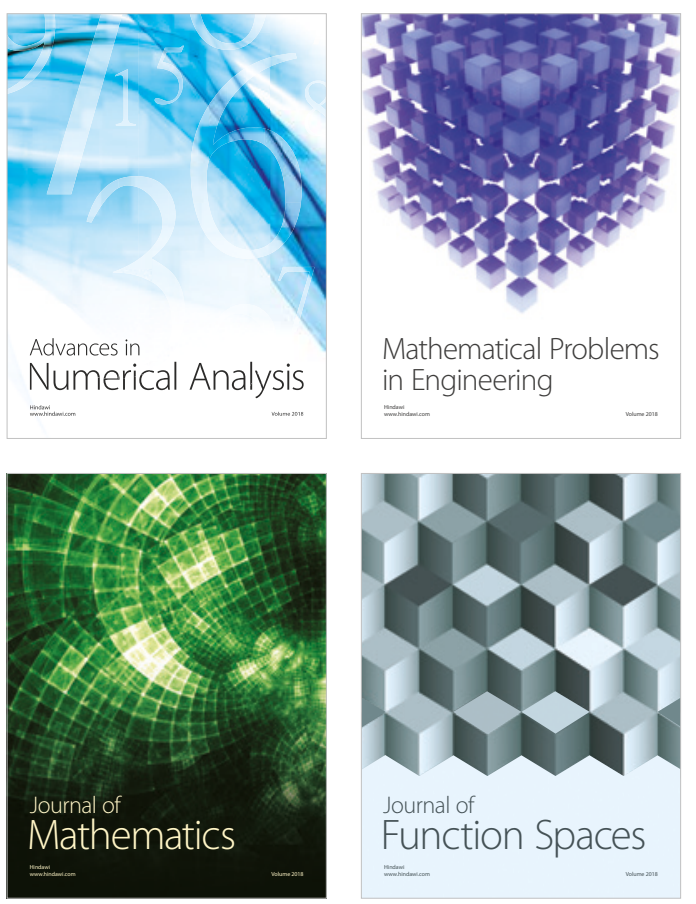

Mathematical Problems in Engineering

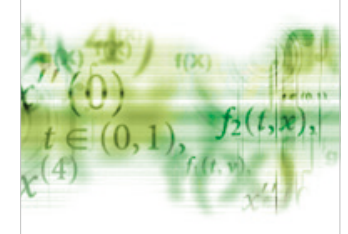

International Journal of

Differential Equations

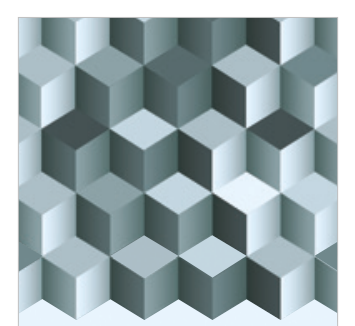

Journal of

Function Spaces
The Scientific

World Journal

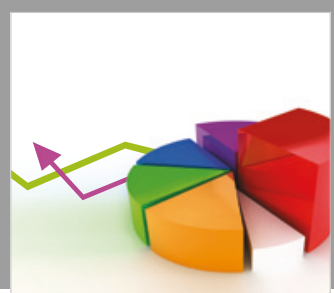

Journal of

Probability and Statistics
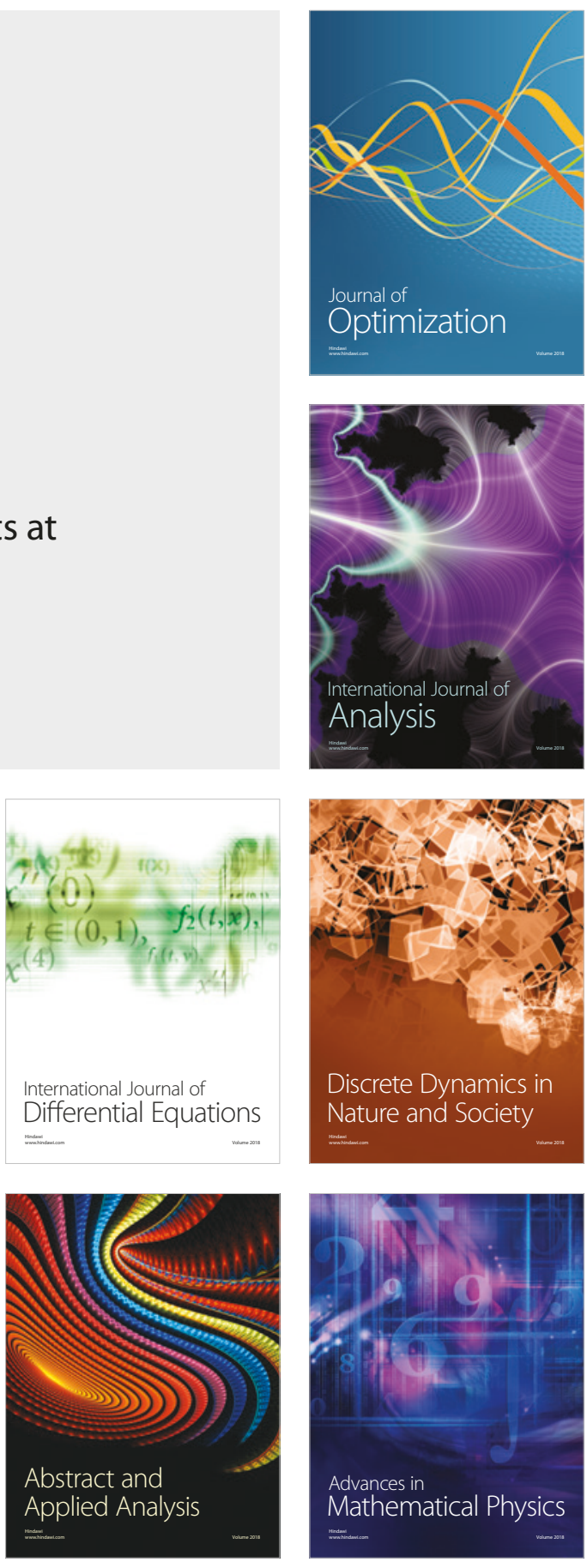